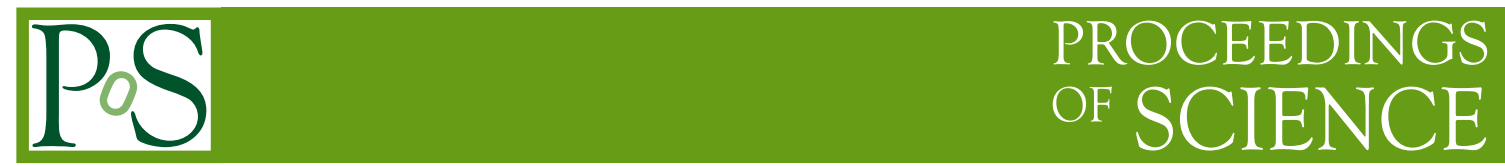

\title{
Introduction to Perturbation Theory
}

\author{
Ciaran Williams* \\ Department of Physics, University at Buffalo \\ The State University of New York, Buffalo, NY 14260-1500, USA \\ E-mail: ciaranwi@buffalo.edu
}

\begin{abstract}
These lectures are designed to provide an introduction to perturbative techniques used to perform calculations for particle colliders. The aim of these lectures is to provide an overview (and starting point for further reading) of topics which are not typically covered as part of standard Quantum Field Theory graduate courses, but are nevertheless vital for modern applications of QFT for collider physics. Specifically the lectures will cover amplitude calculations using on-shell techniques at tree-level and one-loop, and tools for regularization of Infrared divergences at Next-to-Leading Order (NLO) and Next-to-Next-to Leading Order (NNLO).
\end{abstract}

Theoretical Advanced Study Institute Summer School 2018 'Theory in an Era of Data'(TASI2018) 4 - 29 June, 2018

Boulder, Colorado

${ }^{*}$ Speaker. 


\section{Introduction and Motivation}

Now, more so than ever, precision calculations are indispensable for the ongoing experimental program at CERN's Large Hadron Collider (LHC). This is for several reasons, the experimental analyses are maturing, the detectors are now fully calibrated, and data is pouring in. As a result several analyses have already been presented (for a small selection see e.g. [1, 2, 3, 4, 5, 6, 7, 8, 9]) for which the experimental uncertainties are $a$ ) smaller or comparable to the Next-to-Leading Order (NLO) accuracy and $b$ ) clearly demonstrate the inability of NLO predictions to correctly describe the data. The current round of analyses will quickly exploit the advantages of the larger center of mass energy of Run II. If no new resonances are directly produced, (which are clearly visible over the backgrounds), the field will enter a new regime of precision studies. In lieu of any discoveries the primary objectives of Run II and the future High luminosity upgrade (HL-LHC) will be twofold, firstly the properties of the Higgs boson must be established and rigorously tested against the predictions of the Standard Model (SM). Secondly, subtle Beyond the Standard Model (BSM) physics must be searched for and constrained (or discovered). For both of these objectives precision knowledge of the SM (and putative signals) is crucial.

Fortunately, the current lack of existing evidence for BSM physics need not necessarily hold indefinitely over the lifetime of the LHC. It may simply mean that we have to work harder to uncover its subtleties. The discovery of the Higgs boson [10,11] allows us, for the first time, to study the properties of a fundamental scalar. One of the most fascinating features of such a boson is that it allows for a dimension-two renormalizable term in the Lagrangian $|H|^{2}$, which is unique to a scalar field. This term allows for the possibility of portal type interactions with other, as yet undiscovered, fundamental scalars. Once expanded these terms modify self-interactions of the Higgs boson, and hence the shape of the electro-weak symmetry breaking potential. As it currently stands we have no information (beyond the quadratic term) on the shape of this potential, which could have enormous consequences for BSM physics and cosmology. Of course the SM predicts the shape of this potential through the Higgs quartic coupling $\lambda$, which is fully determined as a function of the Higgs mass and vacuum expectation value. The measurement of this coupling (through di-Higgs production) is a long term goal of the HL-LHC and future colliders [12]. Studies of the Higgs boson will also shed insight into the validity of the SM itself. For instance, we are not currently certainly whether the Higgs is $100 \%$ SM like, or masquerading as a SM Higgs-like boson in the alignment limit of a BSM scenario. In any case, given the widespread nature of extended Higgs sectors in BSM theories, it is extremely important to pin down the nature of the Higgs boson by measuring its interactions with the other SM particles as precisely as possible.

A fantastic recent result is the calculation of the Higgs inclusive cross section to Next-toNext-to-Next-to Leading Order accuracy $\left(\mathrm{N}^{3} \mathrm{LO}\right)[13,14]$. This breakthrough calculation has had immediate significant impact in our ability to understand the Higgs boson. Previously the theoretical uncertainty arising from variation of the renormalization and factorization scales was around $10 \%$ (note that this is already around the experimental uncertainty with only Run I data). With the completion of the inclusive cross section, this now drops to around 2\%. Additionally, convergence of independent PDF sets have also significantly reduced the uncertainties estimated from varying the PDF choices $[15,16,17]$, resulting in much upgraded inclusive prediction for Run II and beyond. One of the key advantages of the Run II (and HL-LHC) program is the ability to study the 
Higgs differentially. Going beyond the inclusive cross section allows us to probe more subtle effects which may by obscured inclusively, and will allow for a more efficient means of separating different Higgs production mechanisms. It is therefore crucial that continued progress is made on the theoretical predictions which allow for fully differential properties of the Higgs boson.

It is clear from the emerging experimental analyses that (at least) NNLO predictions are required in order to compare SM predictions to data. Failure to do so can result in a misinterpretation of the data. For instance, the measurement of the $W W$ cross section $[5,9]$ failed to match the NLO prediction, and deviations of around 2 standard deviations were reported. This lead to speculation that the difference may have been caused by light stops $[18,19]$. However, once the NNLO calculation was completed, the discrepancy disappeared [20]. The assumption that the NLO prediction is equivalent to that of the "SM", while widespread, is incorrect. What is crucial is that theoretical and experimental uncertainties are similar sizes (and ideally small). However, we have no systematic way of estimating theoretical uncertainty. Scale variation is typically used, however, again as more NNLO results are becoming known, we are typically finding that the procedures use at NLO are inadequate to estimate the impact of unknown higher order corrections. Therefore it is now clear that for Run II NNLO predictions for cross section measurements and differential studies are essential.

Over the last decade or so, the bottleneck for NNLO computations was the inherent difficulties in regulating the InfraRed (IR) singularities which are copious in different phase spaces, and cancel only after the combination of the various parts of the calculation. Thankfully our ability to perform these complex NNLO calculations has significantly improved over the last few years. This is primarily due to the maturation of existing technologies [21, 22, 23], and the advent of new techniques $[24,25,26]$. One of the most interesting techniques to emerge over the last couple of years is $N$-jettiness slicing [24, 25]. Slicing techniques [27, 28] deal with the IR singularities by separating the phase space volume in to two separate regions. In one region the calculation is free of some (or all) of the IR singularities and is therefore amenable to integration. In the second region an approximation is typically used to calculate the cross section. The approximation is usually controlled by the size of the slicing variable. Unphysical power corrections are typically present, which must be suppressed. For this reason the values of the slicing parameter must be taken as small as possible. In $N$-jettiness slicing the global event shape variable $N$-jettiness [29] is used to separate the two regions. If, for an $N$ jet final state, the $N$-jettiness $\tau_{N}$ is greater than a cutoff $\tau_{N}^{\text {cut }}$ then the calculation can have at most one single un-resolved parton. In this regard it resembles an NLO calculation and can therefore be calculated with NLO tools, such as Catani-Seymour Dipole subtraction [30]. For the region $\tau_{N}<\tau_{N}^{\text {cut }}$, a factorization theorem [31, 32] derived from Soft Collinear Effective Field Theory (SCET) [33, 34, 35, 36, 37] can be used to approximate the calculation. Once $\tau_{N}^{\text {cut }}$ is taken small enough the power corrections become negligible and the calculation obtains NNLO accuracy. The $N$-jettiness slicing approach has been used in several recent calculations [38, 24, 39, 40, 41], and has been implemented into MCFM [42]. These results, in addition to developments by other groups, have resulted in a veritable explosion of recent NNLO results $[43,44,45,46,47,48,49,50,51,52,53,54,55,56]$.

In summary, assuming that no new resonant particle is found over the next year, the next stage of the LHC becomes a precision era. The search for BSM physics will not end, but will become increasingly difficult. Precision calculations are needed to determine the shape of SM backgrounds 
for subtle shape analyses, such as those arising from Effective Field Theory (EFTs) extensions of the SM, anomalous couplings, and broad resonances which may decay semi-invisibly. Precision calculations are also mandatory in the study of the Higgs boson, for a variety of production channels, such that theoretical uncertainty need not limit our understanding of the Higgs and its interactions with the SM particle spectrum.

The aim of these lectures is therefore to introduce the reader to some of the tools which are helpful in constructing precision predictions for LHC physics. Section 2 provides an overview of modern tools for optimized calculation of tree-level amplitudes, focusing on on-shell techniques for color ordered amplitudes. Section 3 then extends the discussion to NLO computations, introducing the reader to unitarity based methods. Section 4 discusses the difficulties associated with IR regularization at both NLO and NNLO, and finally the tools are applied to an example calculation in Section 5.

Finally, I would at this stage like to acknowledge the great historical sets of lectures available on perturbation theory, from which I have borrowed heavily in constructing these notes. I will try to ensure adequate referencing is included throughout the text, but globally I am grateful to Keith Ellis for his lecture notes from various schools (which have been released as a review article on unitarity [57]). John Campbell for his TASI lecture notes from 2011 [58] and the Black Book of QCD [59], and Lance Dixon's notes from this school in 2014 and 1994 [60, 61, 62]. Without these fantastic resources preparing these lectures would have been a daunting challenge. The wise reader would set these lecture notes down, and go back to these more complete and well written works.

\section{The lowest of orders, techniques for computing tree-level diagrams}

We begin by discussing the very first term needed in a perturbative expansion, the Leading Order (or LO). For the vast majority of processes this corresponds to the calculation of tree-level diagrams, which contain no closed loops. However, there is a subtly in that some processes, (of which the most famous is probably $g g \rightarrow H$ ) are possible only via a loop. This is why tree-level and LO are not the same thing, LO always refers to the first non-zero term in the perturbative expansion. Tree-level always means no loops. Anyway for now we'll assume that LO and treelevel are interchangeable and discuss techniques for calculating tree-level processes.

Many of you have probably already come across some of the great work horses in particle physics, the event generators. A key component of these complex codes such as Madgraph [63, 64], Sherpa [65], Alpegn [66], Pythia [67, 68], and Herwig [69, 70], is the ability to calculate matrix elements for an almost arbitrary number of scattering particles [71]. While the calculation of, for instance, $g g \rightarrow g g$ scattering could be completed over a leisurely afternoon. The factorial growth of the number Feynman diagrams means that already calculating $g g \rightarrow 4 g$ scattering is rather unpleasant. By the time we try to calculate something like $g g \rightarrow 7 g$ the number of Feynman diagrams has grown to 559405. Obviously no-one is going to sit down and simplify this.

Its clear that even writing the matrix element for of multi-particle scattering processes is a horrendous computational challenge. How then is it possible for you to summon Madgraph, implement your favorite model and generate cross sections? The answer lies in the underlying structure of the theory which allows us to organize our calculation, and more importantly, recursively calculate tree-level diagrams. 


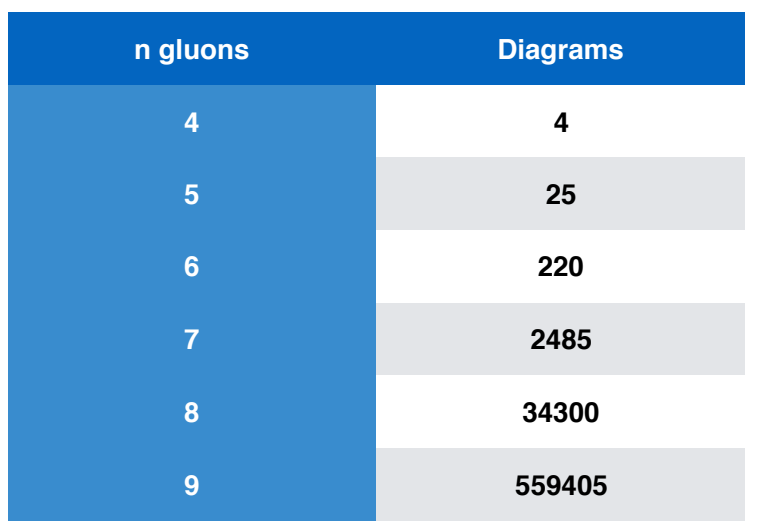

Figure 1: Number of Feynman diagrams for gluonic scattering as a function of the number of external gluons, $n$.

\subsection{Organizing the calculation I : Color}

The basic idea we will pursue is one of divide and conquer. Much of the complexity of the calculation occurs due to the proliferation of color. The more colored scatterers there are, the more different types of color structures we have to calculate. However, since the underlying Feynman diagrams are related to one another, it should be possible to generate different color structures from a single "master" amplitude. These amplitudes go by different names in the literature, we'll call them color-ordered amplitudes here, but you may also see them referred to as partial or primitive amplitudes in other texts. For more detail on these topics you could investigate some of the following review references $[60,61,72]$ and the original literature $[73,74,75,76,77]$.

The key concept of color-decomposition is to systematically remove the structure constants $f^{a b c}$ arising form the Lie Algebra of $S U(N)$ in terms of the generator matrices $T^{a}$ in the fundamental representation. We recall that in the adjoint representation of $S U(N)$ there are $N^{2}-1$ color degrees of freedom, while in the fundamental there are $N$. Gluons of course, transform in the adjoint representation and quarks (anti-quarks) in the fundamental (anti-fundamental) representation. We normalize our matrices as follows

$$
\operatorname{tr}\left(T^{a} T^{b}\right)=\delta^{a b}
$$

This may be different (by a factor of 2) from what you are used to, but will avoid an irritating proliferation of $\sqrt{2}$ 's in calculations. What can happen in a QCD calculation? Well we can either encounter a gluon-quark-anti quark vertex (factor of $\left(T^{a}\right)_{i}^{\bar{j}}$.) a triple gluon vertex (factor of $f^{a b c}$ ), or a four gluon vertex (factors of $f^{a b e} f^{c d e}$ ). We recall that we can relate structure constants to the generators through the Lie Algebra

$$
\left[T^{a}, T^{b}\right]=i \sqrt{2} f^{a b c} T^{c}
$$

We can use this to write a definition of $f^{a b c}$

$$
i \sqrt{2} f^{a b d} T^{d} T^{c}=\left[T^{a}, T^{b}\right] T^{c}
$$


Taking the trace of both sides

$$
\begin{aligned}
i \sqrt{2} f^{a b d} \operatorname{tr}\left(T^{d} T^{c}\right) & =\operatorname{tr}\left(T^{a} T^{b} T^{c}\right)-\operatorname{tr}\left(T^{b} T^{a} T^{c}\right) \\
i \sqrt{2} f^{a b c} & =\operatorname{tr}\left(T^{a} T^{b} T^{c}\right)-\operatorname{tr}\left(T^{b} T^{a} T^{c}\right) \\
i \sqrt{2} f^{a b c} & =\operatorname{tr}\left(T^{a} T^{b} T^{c}\right)-\operatorname{tr}\left(T^{a} T^{c} T^{b}\right)
\end{aligned}
$$

So after making this replacement our amplitude will now contain many products of traces. If external quarks are present there will also be strings of matrices which are terminated by the fundamental indices associated with the quarks, e.g. $\left.\left(T^{a_{1}} \ldots T^{m_{1}}\right)_{i}^{\bar{j}}\right)$. Many of the trace products will have a repeated index e.g. $\operatorname{tr}\left(\ldots T^{a} \ldots\right) \operatorname{tr}\left(\ldots T^{a} \ldots\right)$ (due to the presence of propagating gluons which include $\delta_{a b}$ terms). We can simplify these terms further by using the Fierz identity

$$
\left(T^{a}\right)_{i}^{\bar{j}}\left(T^{a}\right)_{l}^{\bar{k}}=\delta_{i}^{\bar{k}} \delta_{l}^{\bar{j}}-\frac{1}{N} \delta_{i}^{\bar{j}} \delta_{l}^{\bar{k}}
$$

For instance consider a Feynman diagram (shown in Fig. 2) for four gluon scattering consisting of a chain of gluon three-vertices. This will have a color structure proportional to $f^{a_{1} a_{2} b_{1}} f^{b_{1} a_{3} a_{4}}$ where $a_{i}$ are out going gluons and $b_{1}$ is the connecting gluon, after re-writing the $f^{a b c}$ in terms of the generators we have $2^{2}=4$ terms. Each of which look like the product of two traces containing three generators e.g.

$$
\operatorname{tr}\left(T^{a_{1}} T^{a_{2}} T^{b_{1}}\right) \operatorname{tr}\left(T^{b_{1}} T^{a_{3}} T^{a_{4}}\right)+\ldots
$$

Next we apply the Fierz identity and we end up with two traces

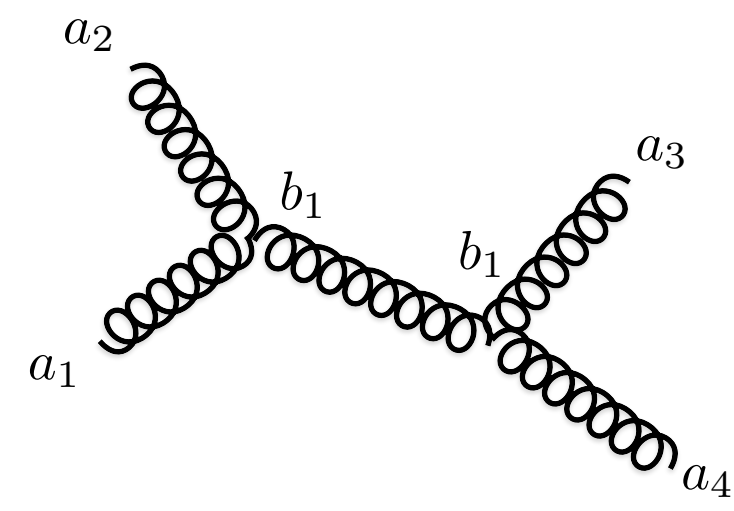

Figure 2: Example topology for a color analysis of a single Feynman diagram.

$$
\operatorname{tr}\left(T^{a_{1}} T^{a_{2}} T^{b_{1}}\right) \operatorname{tr}\left(T^{b_{1}} T^{a_{3}} T^{a_{4}}\right)=\operatorname{tr}\left(T^{a_{1}} T^{a_{2}} T^{a_{3}} T^{a_{4}}\right)-\frac{1}{N} \operatorname{tr}\left(T^{a_{1}} T^{a_{2}}\right) \operatorname{tr}\left(T^{a_{3}} T^{a_{4}}\right)
$$

Now included in our ... in equation 2.8 is a term which has $a_{3}$ and $a_{4}$ swapped, but leaves $a_{1}$ and $a_{2}$ in the same order, this term also comes in with a minus sign with respect to the piece we just 
looked at. As a result the $1 / N_{c}$ terms cancel. We are left only with combinations of single trace terms. This is a generic result for the tree-level scattering of gluons, and accordingly an $n$-gluon amplitude can be written as follows

$$
\mathscr{A}_{n}^{(0), g l u}\left(\left\{p_{i}, \lambda_{i}, a_{i}\right\}\right)=g^{n-2} \sum_{\sigma \in S_{n} / Z_{n}} \operatorname{tr}\left(T^{a_{\sigma(1)}} \ldots T^{a_{\sigma(n)}}\right) A_{n}^{(0)}\left(\sigma\left(1_{1}^{\lambda}\right), \ldots, \sigma\left(n^{\lambda_{n}}\right)\right) .
$$

There is a lot introduced in this equation, so lets go through it a little. Firstly we note the notation $\left\{p_{i}, \lambda_{i}, a_{i}\right\}$ which corresponds to the information about our scattering gluons, namely their individual momentum $p_{i}$, helicity $\lambda_{i}$ and color $a_{i}$. We'll talk more about the helicity shortly, but hopefully momentum and color are by now obvious. On the right hand $S_{n}$ is the set of all permutations of $n$ objects, while $Z_{n}$ is the set of cyclic permutations. Therefore we sum over the set $S_{n} / Z_{n}$ to define all inequivalent orders in the trace. We have therefore factorized color and kinematics. The real work is now in calculating the color-stripped amplitude $A_{n}^{(0)}$, you may initially think that this is not much of a simplification, however since there is a specific allowed permutation of gluons we will be able to infer much about the factorization and pole structure of the amplitude. Which will allow us to construct clever recursion relations for tree-level amplitudes. Finally we mention that having

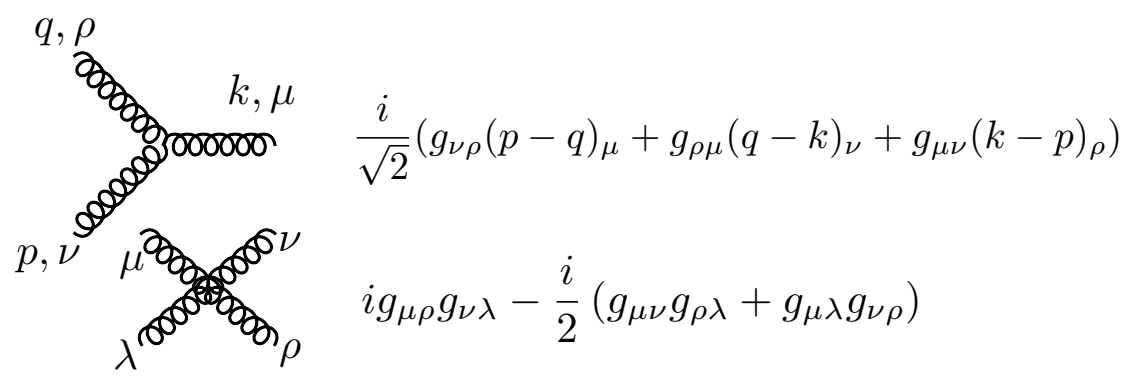

Figure 3: Color ordered Feynman rules for gluon scattering

quarks in the amplitude adds additional complexities, however for a single quark/anti-quark pair the sum can be written simply as follows

$$
\mathscr{A}_{n}^{(0), q \bar{q}}\left(\left\{p_{i}, \lambda_{i}, a_{i}\right\}\right)=g^{n-2} \sum_{\sigma \in S_{n-2}}\left(T^{a_{\sigma(3)}} \ldots T^{a_{\sigma(n)}}\right)_{i_{2}}^{\overline{j_{1}}} A_{n}^{(0)}\left(1_{\bar{q}}^{\lambda_{1}}, 2_{q}^{\lambda_{2}}, \sigma\left(3_{3}^{\lambda}\right), \ldots, \sigma\left(n^{\lambda_{n}}\right)\right)
$$

Where now the sum is over the set of $n-2$ permutations and each amplitude multiples a matrix in color space. Upon stripping of the color factors into the above decomposition we can apply color-ordered Feynman rules, shown for gauge bosons in the figure, which do not have any color information (but require a consistent order of particles). For a more in-depth discussion of color decomposition (and a nice discussion of this topic in general) involving multiple quark pairs can be found in Ref. [78].

In summary we see that the complexities associated with color can be lessened by grouping the pieces of the calculation together into color ordered amplitudes. Although becoming more complicated, the same ideas work at higher orders [77, 78]. Thus we treat the color organization as a solved problem for now, and focus instead on how to go about calculating color ordered amplitudes. 


\subsection{Organizing the calculation II : Helicity}

With color under control we turn our attention next to conquering the kinematics. We will do this using helicity to simplify our calculation. Why is helicity a simplifying feature? Well at all modern colliders our fermions are ultra-relativistic meaning that $|\mathbf{p}| \sim E>>m$ and $p^{2}=0$ is an excellent approximation for every quark bar the top quark (and occasionally the bottom depending on your specific needs). For massless particles helicity is a good quantum number, so we can classify and compute in the helicity basis. In order to fully exploit the helicity structure we'll move away from traditional four-vectors and instead re-write our amplitudes interms of 2-component Weyl-Spinors (which we just refer to as spinors). We construct our spinors out of solutions to the massless Dirac equation

$$
\not p_{i} u_{ \pm}\left(p_{i}\right)=\not p_{i}\left|i^{ \pm}\right\rangle=0
$$

Here $u_{ \pm}\left(p_{i}\right)$ is a right-(left)-handed spinor $u_{ \pm}\left(p_{i}\right)=1 / 2\left(1 \pm \gamma_{5}\right) u\left(p_{i}\right)$ (written in four-component Dirac notation). We would like to construct Lorentz-invariant quantities out of our spinors, and there are two possible scalar structures,

$$
\begin{aligned}
\langle i j\rangle=\left\langle i^{-} j^{+}\right\rangle & =\bar{u}_{-}\left(k_{i}\right) u_{+}\left(k_{j}\right) \\
{[i j]=\left\langle i^{+} j^{-}\right\rangle } & =\bar{u}_{+}\left(k_{i}\right) u_{-}\left(k_{j}\right)
\end{aligned}
$$

These products are anti-symmetric under the exchange of $i$ and $j$.

$$
\langle i j\rangle=-\langle j i\rangle \quad[i j]=-[j i]
$$

You can check with the helicity projection that $\left\langle i^{ \pm} j^{ \pm}\right\rangle$vanish. We can also build the completeness relation

$$
\begin{aligned}
\left|i^{+}\right\rangle\left\langle i^{+}|+| i^{-}\right\rangle\left\langle i^{-}\right| & =\frac{1}{2}\left(1+\gamma_{5}\right) \not p_{i} \frac{1}{2}\left(1-\gamma_{5}\right)+\frac{1}{2}\left(1-\gamma_{5}\right) \not p_{i} \frac{1}{2}\left(1+\gamma_{5}\right) \\
\left|i^{+}\right\rangle\left\langle i^{+}|+| i^{-}\right\rangle\left\langle i^{-}\right| & =\frac{1}{2}\left(1+\gamma_{5}\right) \not p_{i}+\frac{1}{2}\left(1-\gamma_{5}\right) \not p_{i} \\
\left|i^{+}\right\rangle\left\langle i^{+}|+| i^{-}\right\rangle\left\langle i^{-}\right| & =\not p_{i}
\end{aligned}
$$

We can also define the useful, Gordon, Fierz and Schouten identities

$$
\begin{gathered}
2 p_{i}^{\mu}=\left\langle i\left|\gamma^{\mu}\right| i\right] \\
\left\langle i\left|\gamma^{\mu}\right| j\right]\left\langle k\left|\gamma_{\mu}\right| l\right]=2\langle i k\rangle[l j] \\
\langle i j\rangle\langle k l\rangle=\langle i k\rangle\langle j l\rangle+\langle i l\rangle\langle k j\rangle
\end{gathered}
$$

We can use these relations to relate spinor products to more traditional Lorentz invariant produces

$$
s_{i j}=\left(k_{i}+k_{j}\right)^{2}=2 k_{i} k_{j}=\frac{1}{2}\left\langle i\left|\gamma^{\mu}\right| i\right]\left\langle j\left|\gamma^{\mu}\right| j\right]=\langle i j\rangle[j i]
$$


So we see that spinor products behave like square roots of Mandelstam invariants. This is one of the reasons why they are natural quantities to write amplitudes in terms of, since amplitudes themselves are roots of the matrix element squared (which is a function of Mandelstam invariants).

The last quantity we'll need is the spinor representation of a polarization vector $\varepsilon_{\mu}$. This is a little tricker since for a massless gauge boson there is an inherent gauge invariance. This means that the vector is defined with reference to an arbitrary light-like momentum $q$. In terms of this momentum we have the following polarization vectors

$$
\varepsilon_{\mu}^{+}(p, q)=\frac{\left\langle p\left|\gamma_{\mu}\right| q\right]}{\sqrt{2}\langle q k\rangle} \quad \varepsilon_{\mu}^{-}(p, q)=-\frac{\left[p\left|\gamma_{\mu}\right| q\right\rangle}{\sqrt{2}[q k]}
$$

Its easy to see that these vectors are transverse $(\varepsilon \cdot p=0)$ If you wish to test your budding spinor skills you can also show that changing the reference momentum $q \rightarrow q^{\prime}$ results in a shift in $\varepsilon$ which is proportional to $p_{\mu}$, and, as a result has no physical consequence on the amplitude (by the Ward Identity).

2.2.1 An example $e^{+} e^{-} \rightarrow q \bar{q}$

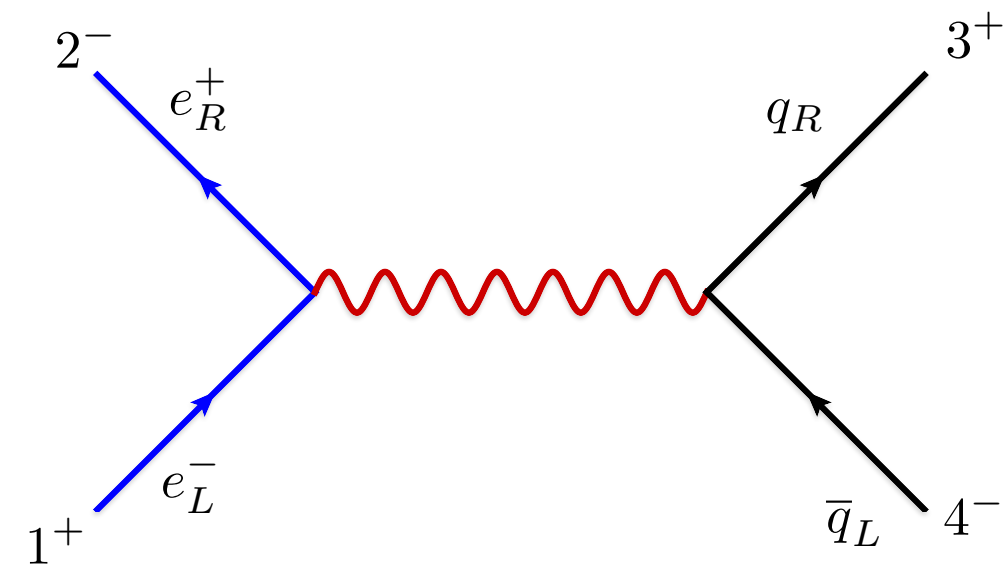

Figure 4: LO Feynman diagram for $e^{+} e^{-} \rightarrow q \bar{q}$ in the helicity basis.

As an example lets think about $e^{+} e^{-} \rightarrow q \bar{q}$. To make our lives easier we'll call the electron $e$ and the positron $\bar{e}$ to avoid confusing electric charge and helicity. We'll calculate the following helicity amplitude

$$
A_{4}^{(0)}\left(1_{\bar{e}}^{+}, 2_{e}^{-}, 3_{q}^{+}, 4_{\bar{q}}^{-}\right)
$$

there is a single Feynman diagram, illustrated in Fig. 4. We'll treat all particles as outgoing, as such the incoming left-handed electron is treated as if it were an out-going right-handed electron (and similarly for the quark). Thus in the traditional notation we have

$$
A_{4}^{(0)}\left(1_{\bar{e}}^{-}, 2_{e}^{+}, 3_{q}^{-}, 4_{\bar{q}}^{+}\right)=\bar{v}_{-}\left(p_{2}\right) \gamma^{\mu} u_{-}\left(p_{1}\right) \frac{g_{\mu v}}{2 s_{12}} \bar{u}_{+}\left(p_{3}\right) \gamma_{v} v_{+}\left(p_{4}\right)
$$


Although not explicitly stated in the previous section to avoid an over abundance of definitions, $v$ spinors can be readily obtained from $u$ ones via the following relationship

$$
u_{ \pm}=v_{\mp}
$$

Using this we can re-write our expression in the spinor basis

$$
A_{4}^{(0)}\left(1_{\bar{e}}^{+}, 2_{e}^{-}, 3_{q}^{+}, 4_{\bar{q}}^{-}\right)=\frac{\left\langle 2\left|\gamma^{\mu}\right| 1\right]\left\langle 4\left|\gamma_{\mu}\right| 3\right]}{2 s_{12}}
$$

Using our spinor identities this becomes

$$
A_{4}^{(0)}\left(1_{\bar{e}}^{+}, 2_{e}^{-}, 3_{q}^{+}, 4_{\bar{q}}^{-}\right)=\frac{\langle 24\rangle[13]}{s_{12}}
$$

At this point we have an expression we could code up into Fortran/C++ and coupled with a phase space integrator we could calculate the cross section for this process. However we can further tidy up this expression as follows

$$
\frac{\langle 24\rangle[13]}{s_{12}}=\frac{\langle 24\rangle[13]}{\langle 12\rangle[21]}=\frac{\langle 24\rangle[13]\langle 34\rangle}{\langle 12\rangle[21]\langle 34\rangle}
$$

Then, using momentum conservation

$$
\frac{\langle 24\rangle[13]\langle 34\rangle}{\langle 12\rangle[21]\langle 34\rangle}=\frac{\langle 24\rangle\langle 4|p / 3| 1]}{\langle 12\rangle[21]\langle 34\rangle}=-\frac{\langle 24\rangle\left\langle 4\left|p_{2}\right| 1\right]}{\langle 12\rangle[21]\langle 34\rangle}=-\frac{\langle 24\rangle\langle 42\rangle[21]}{\langle 12\rangle[21]\langle 34\rangle}
$$

Thus we see that

$$
A_{4}^{(0)}\left(1_{\bar{e}}^{+}, 2_{e}^{-}, 3_{q}^{+}, 4_{\bar{q}}^{-}\right)=\frac{\langle 24\rangle^{2}}{\langle 12\rangle\langle 34\rangle}
$$

The above equation depends only on the $\langle i j\rangle$ spinor products, we call it holomorphic. It is our first encounter of a special type of helicity amplitude in which there are two negative helicity states and an (arbitrary) number of positive helicity states. These amplitudes are called MHV, which stands for maximally helicity violating amplitudes. For this four point amplitude we could have also gone a different way and written it as

$$
A_{4}^{(0)}\left(1_{\bar{e}}^{+}, 2_{e}^{-}, 3_{q}^{+}, 4_{\bar{q}}^{-}\right)=\frac{[13]^{2}}{[12][34]}
$$

This amplitude is anti-holomorphic and is referred to as $\overline{\mathrm{MHV}}$, of course for this amplitude the two are equivalent. At the moment you could be forgiven for thinking that the helicity basis induces no overall simplification at all, since we have only calculated 1 of 16 possible helicity configurations. However the parity and symmetry properties of our massless scatterers mean that we can obtain all remaining helicity amplitudes from the one calculated. First we note that amplitudes of the type $A_{4}^{(0)}\left(1_{\bar{e}}^{+}, 2_{e}^{+}, 3_{q}^{+}, 4_{\bar{q}}^{-}\right)$vanish, (they end up with a term which goes like $\left(1-\gamma_{5}\right)\left(1+\gamma_{5}\right)$ ). This leaves a grand total of 4 amplitudes to compute. They correspond to flipping the helicity of the leptons and quarks. We can relate these helicity flips to conjugation and parity operations on our existing amplitude. For example operating on our amplitude with charge conjugation on the lepton 
line exchanges labels 1 and 2, which can be thought of as flipping the helicities of our original amplitude e.g.

$$
A_{4}^{(0)}\left(1_{\bar{e}}^{-}, 2_{e}^{+}, 3_{q}^{+}, 4_{\bar{q}}^{-}\right)=A_{4}^{(0)}\left(1_{e}^{+}, 2_{\bar{e}}^{-}, 3_{q}^{+}, 4_{\bar{q}}^{-}\right)=A_{4}^{(0)}\left(1_{\bar{e}}^{+}, 2_{e}^{-}, 3_{q}^{+}, 4_{\bar{q}}^{-}\right){ }_{1 \leftrightarrow 2}=-\frac{\langle 14\rangle^{2}}{\langle 12\rangle\langle 34\rangle}
$$

We could use the conjugation operator to get all of the amplitudes, but its also useful for us to introduce parity at this stage too. The Parity operation exchanges all of the helicity quantum numbers and thus changes $\langle i j\rangle \leftrightarrow[i j]$. For instance

$$
A_{4}^{(0)}\left(1_{\bar{e}}^{-}, 2_{e}^{+}, 3_{q}^{-}, 4_{\bar{q}}^{+}\right)=A_{4}^{(0)}\left(1_{\bar{e}}^{+}, 2_{e}^{-}, 3_{q}^{+}, 4_{\bar{q}}^{-}\right)_{<>\leftrightarrow[}
$$

So by combining conjugation and parity operations on helicity amplitudes we can obtain many new amplitudes from our existing calculation.

\subsection{Computing arbitrary matrix elements at LO}

We've seen how to organize a general multi-parton calculation in QCD, now we'll look at some ways of calculating arbitrary processes.

\subsubsection{On-shell recursion, the $\mathrm{BCFW}$ relation}

We begin by investigating on-shell recursion techniques, originally discovered by Britto, Cachazo and Feng [79] (and then proven in generality with Witten [80]). This proof is a beautiful result which relies on some Complex analysis and the simple factorization properties of tree-level results. The BCFW recursion relations hence represent a remarkably simple yet deep approach to the calculation of tree amplitudes in gauge theories. Essentially they show that the entire spectrum of tree amplitudes can be calculated in a theory from knowledge of the three point vertices alone. The proof is remarkably simple and relies only on complex analysis and the universal factorization of tree amplitudes. There has been a huge range of applications of the recursion relations, for instance, from formal developments in QFT [81, 82, 83] to phenomenological applications in QCD [84, 85]. Here we sketch the details of the proof. One begins by taking an on-shell amplitude (for now we consider massless particles in the final state) $A_{n}^{(0)}$ and selecting two of the partons $p_{i}$ and $p_{j}$ for special treatment. We wish to shift these momenta such that overall momentum conservation is retained

$$
p_{i}^{\mu} \rightarrow p_{i}^{\mu}+\frac{z}{2}\left\langle j\left|\gamma^{\mu}\right| i\right] \quad p_{j}^{\mu} \rightarrow p_{j}^{\mu}-\frac{z}{2}\left\langle j\left|\gamma^{\mu}\right| i\right]
$$

We note that $p_{i}(z)+p_{j}(z)=p_{i}+p_{j}$ such that local momentum conservation is clearly preserved. Furthermore

$$
p_{i}^{2}(z)=\left(p_{i}^{\mu}+\frac{z}{2}\left\langle j\left|\gamma^{\mu}\right| i\right]\right)\left(p_{\mu, i}+\frac{z}{2}\left\langle j\left|\gamma_{\mu}\right| i\right]\right)=0
$$

which implies that $p_{i}(z)$ is also on-shell. The shifts we have made are equivalent to the following spinor shifts,

$$
\begin{gathered}
|i\rangle \rightarrow|i\rangle+z|j\rangle \\
\mid j] \rightarrow \mid j]-z \mid i]
\end{gathered}
$$




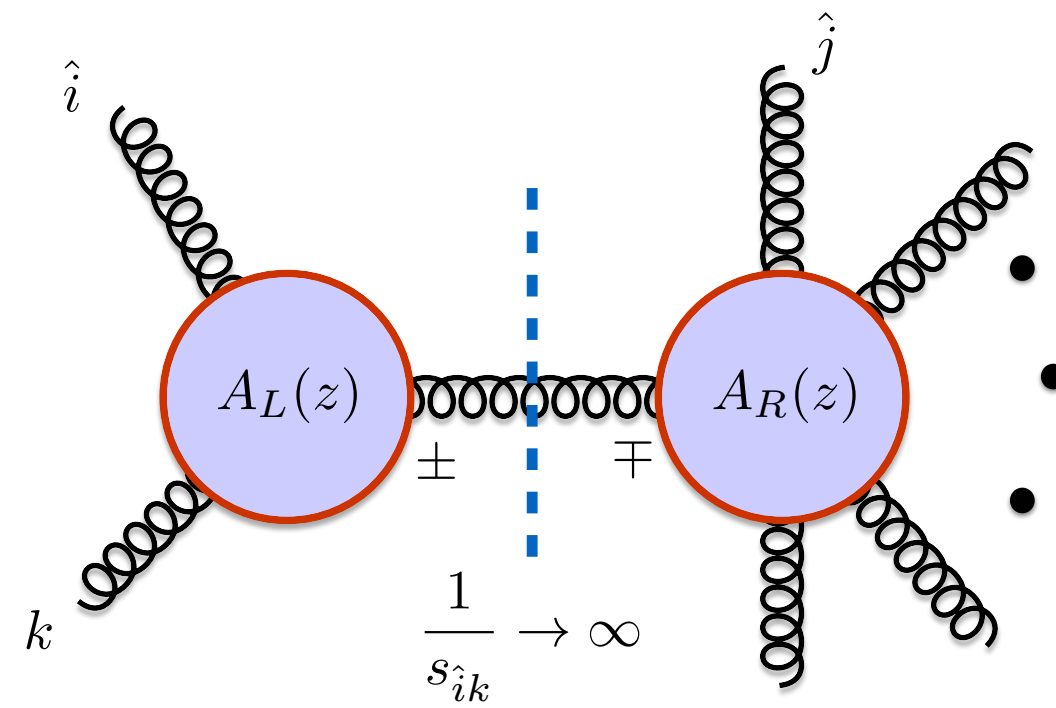

Figure 5: Residues in a tree-level Feynman diagram can only occur when an internal propagator goes onshell resulting in the factorization into two on-shell tree amplitudes.

whilst leaving $\mid i]$ and $|j\rangle$ unaltered. This breaking of symmetry between angle and square brackets breaks a conjugation symmetry for our momentum vector $p_{i}(z)$. As a result the momentum $p_{i}(z)$ is no longer a real Minkoswki momentum, but instead is complex. Under our shift our amplitude $A_{n}^{(0)}(\ldots, i, \ldots, j)$ becomes a function of the shifted variable $z$. i.e. $A_{n}^{(0)} \rightarrow A_{n}^{(0)}(z)$. Although now a function of the complex variable $z$, the shifted amplitude bears many similarities to the physical amplitude. Namely momentum conservation and on-shell final states. We note that since the tree level amplitude contains no functions with branch cuts $A_{n}^{(0)}(z)$ is a rational function in $z$. The singular structure of the amplitude $A_{n}^{(0)}(z)$ is completely determined by simple poles in $z$. This can be seen by considering how poles of tree-level amplitudes occur. Tree-level amplitudes can only develop a pole when an internal propagator goes on-shell i.e. $P_{a b c}^{2} \rightarrow 0$, where $P_{a b c}$ is some generic combination of external momenta. Since propagators are quadratic one may expect that double poles in $\mathrm{z}$ could arise, however if the propagator $P_{\alpha \ldots \beta}$ goes on-shell and $i \in\{\alpha, \ldots, \beta\}$, $j \notin\{\alpha, \ldots, \beta\}$ then we have,

$$
P_{\alpha \ldots \beta}^{2}(z)=\left(p_{\alpha}^{\mu}+\cdots+p_{i}^{\mu}+\frac{z}{2}\left\langle j\left|\gamma^{\mu}\right| i\right]+\cdots+p_{\beta}^{\mu}\right)^{2}=P_{\alpha \ldots \beta}^{2}+z\left\langle j\left|P_{\alpha \ldots \beta}\right| i\right]
$$

Which clearly can only have a pole if

$$
z=z_{i}=-\frac{P_{\alpha \ldots \beta}^{2}}{\left\langle j\left|P_{\alpha \ldots \beta}\right| i\right]}
$$

and the lack of a $z^{2}$ term ensures the presence of only simple poles. On the other hand If both $i \in\{\alpha, \ldots, \beta\}, j \in\{\alpha, \ldots, \beta\}$ then the $z$ dependence drops out through the momentum conservation argument discussed above. 
Now that we know the amplitude is a rational function with simple poles so we can write its Laurent series as follows

$$
A_{n}(z)=\sum_{i} \frac{c_{i}}{z-z_{i}}+\sum_{k=0}^{m} d_{k} z^{k}
$$

Since we know that there is some maximum power of $|i\rangle$ and $\mid j]$ in the amplitude we can truncate the series at $m$. Now assume we can choose a shift with the properties that $A_{n}^{(0}(z) \rightarrow 0$ as $z \rightarrow \infty$. This is by no means guaranteed for all possible shifts of pairs of external momentum, but in general it is possible to find at least one shift which has this property. Now in this case $d_{k}=0$ for all $k$ and

$$
A_{n}(z)=\sum_{i} \frac{c_{i}}{z-z_{i}}
$$

what we ultimately want is to calculate the physical amplitude $A_{n}^{(0)}(0)$ which is given by

$$
A_{n}(0)=-\sum_{i} \frac{c_{i}}{z_{i}}
$$

So all we have to do is classify the possible poles induced by our shift $z_{i}$, and their associated residues $c_{i}$. This is where the nice factorization properties of tree-level amplitudes come into play. For a specific residue $z_{i}$ we can constrain the possible topology via factorization. Firstly we know that every external leg as well as the internal propagator $P_{\alpha \ldots \beta}$ is on-shell. Secondly we know that momentum is conserved amongst the sub-set of particles, i.e.

$$
p_{\alpha}+\ldots p_{\beta}+P_{\alpha \ldots \beta}(z)=0
$$

as such the subset of external particles $\alpha, \ldots \beta$ plus the on-shell propagator leg $P_{\alpha \ldots \beta}$ form a physical scattering amplitude with a subset of the external momentum. Exactly the same argument holds for the other subset of momentum (which included $p_{j}$ in our discussion), so both sub sets of particles form scattering amplitudes, with fewer external momenta then the total amplitude. So in this limit our amplitude can be written as

$$
\sum_{h= \pm} \frac{A_{L}^{h}(z) A_{R}^{-h}(z)}{P^{2}(z)}
$$

here $A_{L}$ represents the particles on the "left" side (i.e. $\{\alpha, i \ldots, \beta\}$ and $P_{\alpha, \beta}$ in our example) and $A_{R}$ represents the remaining particles on the "right" hand side. Since the internal propagator could have either positive or negative helicity we sum over possible states, denoted by $h$. We can readily map this onto our Laurent series by writing

$$
P^{2}(z)=\left\langle j\left|P_{\alpha \ldots \beta}\right| i\right]\left(z-z_{i}\right)
$$

such that

$$
c_{i}=\frac{A_{L}^{h}\left(z_{i}\right) A_{R}^{-h}\left(z_{i}\right)}{\left\langle j\left|P_{\alpha \ldots \beta}\right| i\right]}
$$


To obtain the full amplitude we thus have to sum over all possible residues,

$$
A_{n}(z)=\sum_{i} \sum_{h= \pm} \frac{A_{L}^{h}\left(z_{i}\right) A_{R}^{-h}\left(z_{i}\right)}{\left\langle j\left|P_{\alpha \ldots \beta}\right| i\right]\left(z-z_{i}\right)}=\sum_{i} \sum_{h= \pm} \frac{A_{L}^{h}\left(z_{i}\right) A_{R}^{-h}\left(z_{i}\right)}{P_{\alpha . . . . \beta}^{2}(z)}
$$

The physical amplitude we seek is then given by

$$
A_{n}(0)=\sum_{i} \sum_{h= \pm} \frac{A_{L}^{h}\left(z_{i}\right) A_{R}^{-h}\left(z_{i}\right)}{P_{\alpha . . . . \beta}^{2}}
$$

This is the BCFW recursion relation. We note that when maximally applied it tells us that any tree-level scattering amplitude can be constructed solely from three-point vertices. At the cost of introducing complex momentum. Before moving on lets have a little example so you can see them in action .

2.3.2 Example : $e^{+} e^{-} \rightarrow q \bar{q} g$.
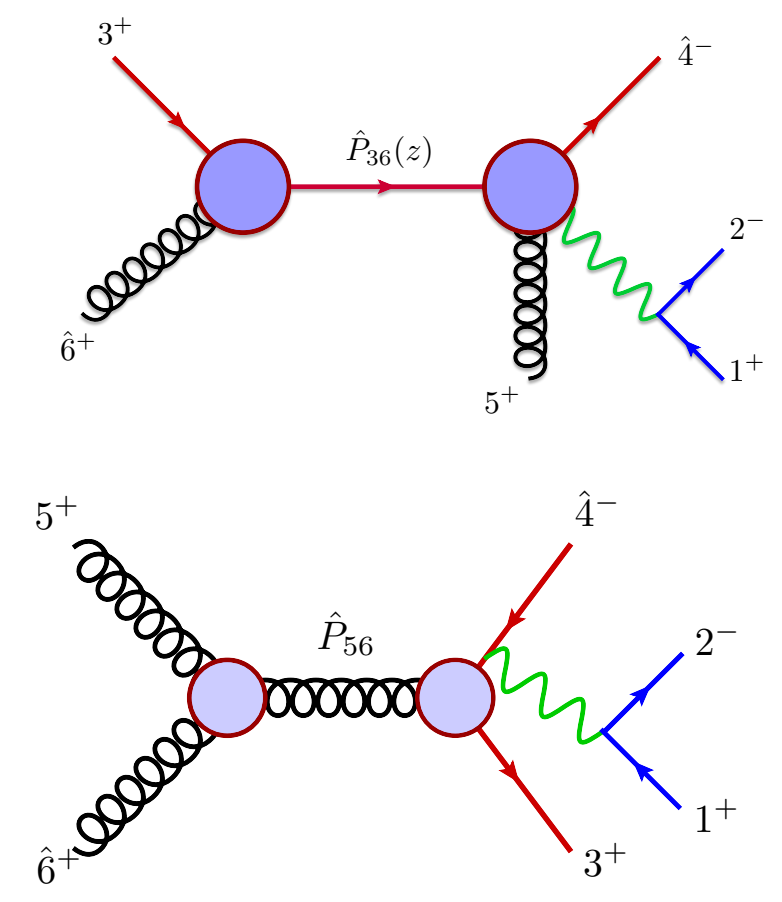

Figure 6: Diagrams which we have to compute given for our chosen shift in our BCFW example calculation.

As an example lets compute the following amplitude

$$
A_{5}^{(0)}\left(1_{\bar{e}}^{+}, 2_{e}^{-}, 3_{q}^{+}, 4_{\bar{q}}^{-}, 5_{g}^{+}\right)
$$

We recall that the four point amplitude is given by the following

$$
A_{4}^{(0)}\left(1_{\bar{e}}^{+}, 2_{e}^{-}, 3_{q}^{+}, 4_{\bar{q}}^{-}\right)=\frac{\langle 24\rangle^{2}}{\langle 12\rangle\langle 34\rangle}
$$


Additionally we'll need the following three-point vertices from QCD

$$
A_{3}^{(0)}\left(1_{q}^{+}, 2_{\bar{q}}^{-}, 3_{g}^{+}\right)=\frac{[13]^{2}}{[12]}
$$

We'll shift the negative helicity quark $\left(p_{4}\right)$ and gluon. We'll delay specifying exactly which shift until we've seen a consequence of complex momentum. Regardless of which shift we choose there is only one possible diagram, with the gluon and un-shifted quark on the left-hand side defining

$$
A_{5}^{(0)}\left(1_{\bar{e}}^{+}, 2_{e}^{-}, 3_{q}^{+}, 4_{\bar{q}}^{-}, 5_{g}^{+}\right)=A_{3}^{(0)}\left(3_{q}^{+}, \hat{5}_{g}^{+}, \hat{P}_{35}^{-}\right) \frac{1}{s_{35}} A_{4}^{(0)}\left(1_{\bar{e}}^{+}, 2_{e}^{-},-\hat{P}_{35}^{+}, \hat{4}_{\bar{q}}^{-}\right)
$$

Lets look at the consequences of complex momenta, momentum conservations tells us that

$$
\left(p_{\hat{5}}+p_{3}\right)^{2}=0 \Longrightarrow p_{\hat{5}} \cdot p_{3}=0
$$

Or

$$
\langle\hat{5} 3\rangle[\hat{5} 3]=0
$$

A spinor product $\langle i j\rangle=0$ implies that $i \propto j$, that is $i$ and $j$ are collinear. For real momentum $|i\rangle$ and $\mid i]$ are related by conjugation, so $\langle i j\rangle=0 \Longrightarrow[i j]=0$. Therefore for real momentum all 3-point vertices of massless momentum vanish. However for complex momentum we can have the case where $\langle i j\rangle=0$ and $[i j] \neq 0$, or vice versa. What does this mean for us? Well we know that in our amplitude we will obtain factors of [35]. We would like this one to be non-zero. This implies that we should shift the negative helicity spinor and leave the positive helicity spinor alone. If we had done the alternative then we would have found that we have no residues (since the amplitude would vanish), but we would have a large $-z$ behavior which would invalidate our recursion. However by choosing

$$
\begin{aligned}
|5\rangle \rightarrow|5\rangle+z|4\rangle & =|\hat{5}\rangle \\
\mid 4] \rightarrow \mid 4]-z \mid 5] & =\mid \hat{4}]
\end{aligned}
$$

This allows us to set $\langle\hat{5} 3\rangle=0$, and have $[\hat{5} 3]=[53] \neq 0$. We can now evaluate our amplitude

$$
A_{5}^{(0)}\left(1_{\bar{e}}^{+}, 2_{e}^{-}, 3_{q}^{+}, 4_{\bar{q}}^{-}, 5_{g}^{+}\right)=\frac{[3 \hat{5}]^{2}}{[3 \hat{P}]} \frac{1}{s_{35}} \frac{\langle 2 \hat{4}\rangle^{2}}{\langle 12\rangle\langle 4(-\hat{P})\rangle}
$$

Inserting our definitions, this can be re-written as ${ }^{1}$

$$
A_{5}^{(0)}\left(1_{\bar{e}}^{+}, 2_{e}^{-}, 3_{q}^{+}, 4_{\bar{q}}^{-}, 5_{g}^{+}\right)=\frac{[35]^{2}}{\langle 4|\hat{P}| 3]} \frac{1}{\langle 35\rangle[53]} \frac{\langle 24\rangle^{2}}{\langle 12\rangle}
$$

We can simplify this further by recalling that

$$
\hat{P}_{35}^{\mu}=P_{35}^{\mu}+\frac{z}{2}\left\langle 4\left|\gamma^{\mu}\right| 5\right] \Longrightarrow\langle 4|\hat{P}| 3]=\left\langle 4\left|P_{35}\right| 3\right]=\langle 45\rangle[53]
$$

\footnotetext{
${ }^{1}$ The astute of you may worry about what happens with the minus sign inside of the spinor product in this step. When I reverse the sign of a Lorentz four vector this is equivalent to multiplying the spinors by a factor of $i$, I have been overall sloppy with my factors of $i$, which should be included every time I have a sub-amplitude, therefore we simply drop this $i$ under the assumption that every amplitude should have an associated $i$ dropped for notational connivence.
} 
So

$$
A_{5}^{(0)}\left(1_{\bar{e}}^{+}, 2_{e}^{-}, 3_{q}^{+}, 4_{\bar{q}}^{-}, 5_{g}^{+}\right)=\frac{\langle 24\rangle^{2}}{\langle 12\rangle\langle 35\rangle\langle 54\rangle}
$$

and we're done. An interesting way of re-writing this amplitude, which will be useful when we discuss factorization later on is

$$
A_{5}^{(0)}\left(1_{\bar{e}}^{+}, 2_{e}^{-}, 3_{q}^{+}, 4_{\bar{q}}^{-}, 5_{g}^{+}\right)=\frac{\langle 34\rangle}{\langle 35\rangle\langle 54\rangle}\left(\frac{\langle 24\rangle^{2}}{\langle 12\rangle\langle 34\rangle}\right)
$$

where we recognize the term in brackets as the four-point amplitude.

We can readily extend our analysis here to calculate the 6-point amplitude as follows. All we need to know in addition to our current knowledge is that in QCD amplitudes of following form vanish

$$
A_{n}^{(0)}\left(1_{q}^{-}, 2_{\bar{q}}^{+}, 3^{+}, \ldots, n^{+}\right)=0
$$

i.e. amplitudes which contain a single quark anti-quark pair and any number of positive helicity gluons (where for us using complex momentum $n \geq 1$ ). As a result when we extend our recursion to the 6 point amplitude we only have two terms to evaluate, which are presented in Fig. 6 . That is we can define the amplitude as the sum of two terms

$$
A_{6}^{(0)}\left(1_{\bar{e}}^{+}, 2_{e}^{-}, 3_{q}^{+}, 4_{\bar{q}}^{-}, 5_{g}^{+}, 6_{g}^{+}\right)=\mathscr{D}_{1}+\mathscr{D}_{2}
$$

The first diagram is similar in structure to our 5-point example above,

$$
\mathscr{D}_{1}=A_{3}^{(0)}\left(3_{q}^{+}, \hat{6}_{g}^{+}, \hat{P}_{36}^{-}\right) \frac{1}{s_{36}} A_{5}^{(0)}\left(1_{\bar{e}}^{+}, 2_{e}^{-},-\hat{P}_{36}^{+}, \hat{4}_{\bar{q}}^{-}, 5_{g}^{+}\right)
$$

here we have shifted $p_{6}$ rather than $p_{5}$ but that is of little consequence. We could follow exactly the same steps as before finding the single diagram gives

$$
\mathscr{D}_{1}=\frac{\langle 24\rangle^{2}[63]}{\langle 12\rangle\langle 36\rangle\langle 45\rangle\langle 5|\hat{P}| 3]}
$$

Inserting our definition for $\hat{P}$ we see that

$$
\mathscr{D}_{1}=\langle 5|\hat{P}| 3]=\langle 56\rangle[63]+z\langle 54\rangle[63]
$$

Now we have to solve for $z$ explicitly, using the formula from the previous section

$$
z=\frac{\langle 36\rangle}{\langle 34\rangle}
$$

and we have

$$
\mathscr{D}_{1}=\frac{\langle 24\rangle^{2}}{\langle 12\rangle\langle 36\rangle\langle 45\rangle(\langle 36\rangle\langle 45\rangle+\langle 34\rangle\langle 56\rangle)}
$$

We use the ultra useful Schouten identity to write this in the compact form

$$
\mathscr{D}_{1}=\frac{\langle 24\rangle^{2}\langle 34\rangle}{\langle 12\rangle\langle 35\rangle\langle 36\rangle\langle 45\rangle\langle 56\rangle}
$$


Turning to the second diagram and using our known amplitudes we see that

$$
\mathscr{D}_{2}=A_{3}^{(0)}\left(5_{g}^{+}, \hat{6}_{g}^{+}, \hat{P}_{56}^{-}\right) \frac{1}{s_{56}} A_{5}^{(0)}\left(1_{\bar{e}}^{+}, 2_{e}^{-},-\hat{P}_{56}^{+}, \hat{4}_{\bar{q}}^{-}, 3_{g}^{+}\right)
$$

The three gluon $\overline{\mathrm{MHV}}$ amplitude is given by

$$
A_{3}^{(0)}\left(1_{g}^{+}, 2_{g}^{+}, 3_{g}^{-}\right)=\frac{[12]^{3}}{[23][31]}
$$

Which allows to define $\mathscr{D}_{2}$ as follows,

$$
\mathscr{D}_{2}=\frac{-\langle 24\rangle^{2}}{\langle 12\rangle\left(z_{56}\langle 34\rangle+\langle 36\rangle\right)\langle 45\rangle\langle 56\rangle}
$$

and here the solution for a residue in $z$ occurs when

$$
z_{56}=\frac{\langle 56\rangle}{\langle 45\rangle}
$$

which allows us to write

$$
\frac{-\langle 24\rangle^{2}}{\langle 12\rangle\left(z_{56}\langle 34\rangle+\langle 36\rangle\right)\langle 45\rangle\langle 56\rangle}=\frac{-\langle 24\rangle^{2}}{\langle 12\rangle\langle 35\rangle\langle 46\rangle\langle 56\rangle}
$$

Putting the two terms together to obtain the total amplitude (after one further application of the Schouten identity) we see that

$$
A_{6}^{(0)}\left(1_{\bar{e}}^{+}, 2_{e}^{-}, 3_{q}^{+}, 4_{\bar{q}}^{-}, 5_{g}^{+}, 6_{g}^{+}\right)=\frac{\langle 24\rangle^{2}}{\langle 12\rangle\langle 45\rangle\langle 56\rangle\langle 63\rangle}
$$

We have thus seen how the BCFW recursion relations can be used to quickly compute a 6 point amplitude. An advantage of all recursion relations is how readily they can be implemented numerically. This allows for rapid generation of high multiplicity final state amplitudes from the working knowledge of lower point amplitudes. This section has introduced two of the key concepts which allows for generation of arbitrarily high multiplicity final state processes at the LHC, color decomposition and recursion. By cleverly applying theses tools modern generators can produce in a reasonable time, a LO cross section for any process of phenomenological interest at the LHC. In the next section we will address how to go beyond LO. Increased precision is mandatory, since it has been repeatedly demonstrated that a LO prediction in QCD is woefully inadequate of describing data.

\section{Next-to-Leading Order computations}

\subsection{Introduction}

Thus far we've focussed our attention on how to efficiently construct tree-level amplitudes. We are now ready to turn our attention to the next order in perturbation theory, corresponding to Nextto-Leading Order (NLO) computations. Over the last 10 years our tools for NLO computations have become so advanced that essentially any process relevant for the LHC program can be computed 
at this accuracy. At the heart of this revolution were the new techniques inspired by unitarity cuts. All modern one-loop generators $[86,87,88,71]$ use methods based upon these techniques to some extent.

The most obvious difference for NLO computations with respect to tree-level counterparts is the presence of loop-diagrams. Loop-diagrams by construction contain a unconstrained momentum, which must be integrated over. Compare to tree-level scattering the complexity of the integrals is significantly increased. As the number of closed loops increases the complexity does also. In general the approach is to reduce the amplitude from a generic expansion to a smaller set of Master Integrals (MI's). As the number of external particles increases, the difficulty in the reduction does too. After reduction the MI's themselves have to be evaluated. It is here that new complications arise. Most higher order loop diagrams contain multiple divergences, both at high values of the loop momentum $(\ell \rightarrow \infty)$ known as Ultraviolet (UV) divergences, and at low values of the loop momentum $(\ell \rightarrow 0)$ known as Infrared (IR) divergences. Both must be regulated, and typical are done so using dimensional regularization (in which the number of spacetime dimensions is altered from four to $d=4-2 \varepsilon$, the IR and UV poles thus emerge as inverse powers of $\varepsilon$. Of two types of poles those attributed to IR physics are much more complicated when attempting to construct a Monte Carlo code designed to make predictions for differential distributions at the LHC. This is because the IR singularities are spread across different phase spaces, and therefore the cancellation of the singularities is far more intricate then the analytic operation of renormalisation, which handles the UV divergences.

The aims for these lecture notes are thus three-fold

- Introduce unitarity techniques, such that you can have a basic understanding of what goes into modern one-loop generators.

- Introduce IR factorization principles

- Demonstrate the cancellation of IR poles in a Monte Carlo using a subtraction approach.

I'll assume that you have all been introduced to renormalization in your graduate lectures, and therefore not focus too much on these aspects. Ideally at the end of these lectures you will have a good understanding of the physics behind NLO generators. This section will introduce and discuss unitarity methods, while the next will focus on the complications arising from IR singularities.

\subsection{Virtual Corrections}

For a process which is non-zero at tree-level there will be two types of corrections which enter at NLO. These correspond to two types of topologies. Since each vertex brings a factor of $g$ we require two vertices to obtain our $\mathscr{O}(\alpha) \propto g^{2}$ correction. The two types of topologies thus correspond to the two possible ways of connecting the new vertices. Firstly we could dress a single tree-level diagram with two vertices. Since this has to interfere with the original tree-level process its clear that the two additional vertices must be ultimately connected such that no new final state particles enter the correction. Thus we are describing a correction which has a closed loop topology. On the other hand, if we dress the diagram with a single emission, we collect one additional factor of $g$ and one additional final state particle, this can then interfere with itself, obtaining $g^{2}$ in the 
sum. Since these topology contains a new final state particle, it modifies the kinematics of the LO topology. These topologies are thus referred to as "real" (the loop topologies are known as virtual, since the emission never exists as a final state particle). In this section we will focus on

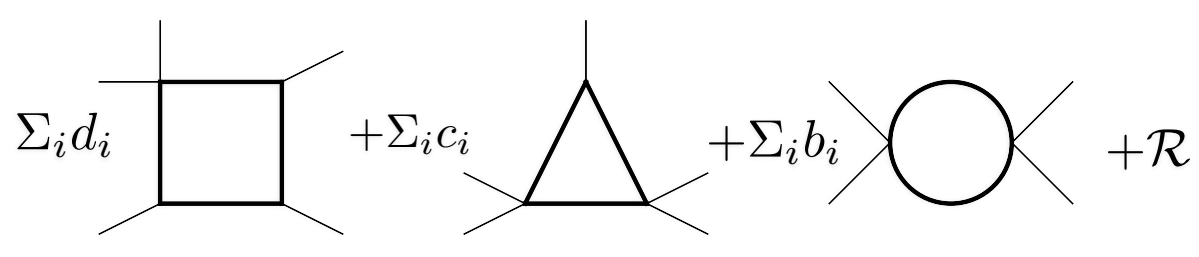

Figure 7: A basis for one-loop amplitudes in terms of box, triangle and bubble integrals and a rational function. The coefficients are rational function of the external momentum.

virtual corrections, leaving the real corrections to the next section. At one-loop it was shown in the mid-90s that an arbitrary multi-particle final state can be written in the following basis [89]

$$
A_{n}^{(1)}=\sum_{i} d_{i} I_{4}^{i}+\sum_{j} c_{j} I_{3}^{j}+\sum_{k} b_{k} I_{2}^{k}+\mathscr{R}
$$

In the above equation $I_{n}^{i}$ represents an $n$-propagator scalar loop integral. For example $I_{4}$ corresponds to a box-integral with four propagators

$$
I_{4}^{P_{1}, P_{2}, P_{3}}=\int \frac{d^{d} \ell}{(2 \pi)^{d}} \frac{1}{\ell^{2}\left(\ell-P_{1}\right)^{2}\left(\ell-P_{2}\right)^{2}\left(\ell-P_{3}\right)^{2}}
$$

Where $P_{i}$ is some combination of external momentum, and the summation over $i$ corresponds to all possible ways of assigning the external states to a box topology. In the above formula's we've implicitly (and explicitly) presumed that the internal particles as massless. Massive particles in the loop also contribute tadpole terms $\sum a_{i} I_{1}^{i}$. The term $\mathscr{R}$ denotes a piece which is a rational function of the external invariants. The coefficients $d c$ etc. are all rational functions of the external momentum and do not depend on the loop momentum. Pictorially this basis is illustrated in Fig. 7.

At first glance, the decomposition into the above basis seems quite remarkable. Since in general we would expect an $n$-point loop integral to be present for a process with $n$ particles in the final state. Such Feynman diagrams to appear at the loop level, of course, but as was shown in Ref. [89] these pentagon and higher point integrals can be reduced down into this basis. The proof of this result is beyond the scope of these lectures but can be found in the original reference. The only assumption we made is that we require an expansion accurate to $\mathscr{O}\left(\varepsilon^{0}\right)$ and that our external states live if four-dimensions. If an all orders result in the regulating parameter $\varepsilon$ is required, a more complicated basis which includes pentagons and higher dimensional boxes, triangle and bubbles (but no rational part) can be used. However for our purposes the above basis is fine.

\subsubsection{Unitarity cuts, an introduction}

Now that we have a basis our next step is to try to construct a tactic to isolate the coefficients of each term. Once we've done that our one-loop process is completely solved. At the heart of 


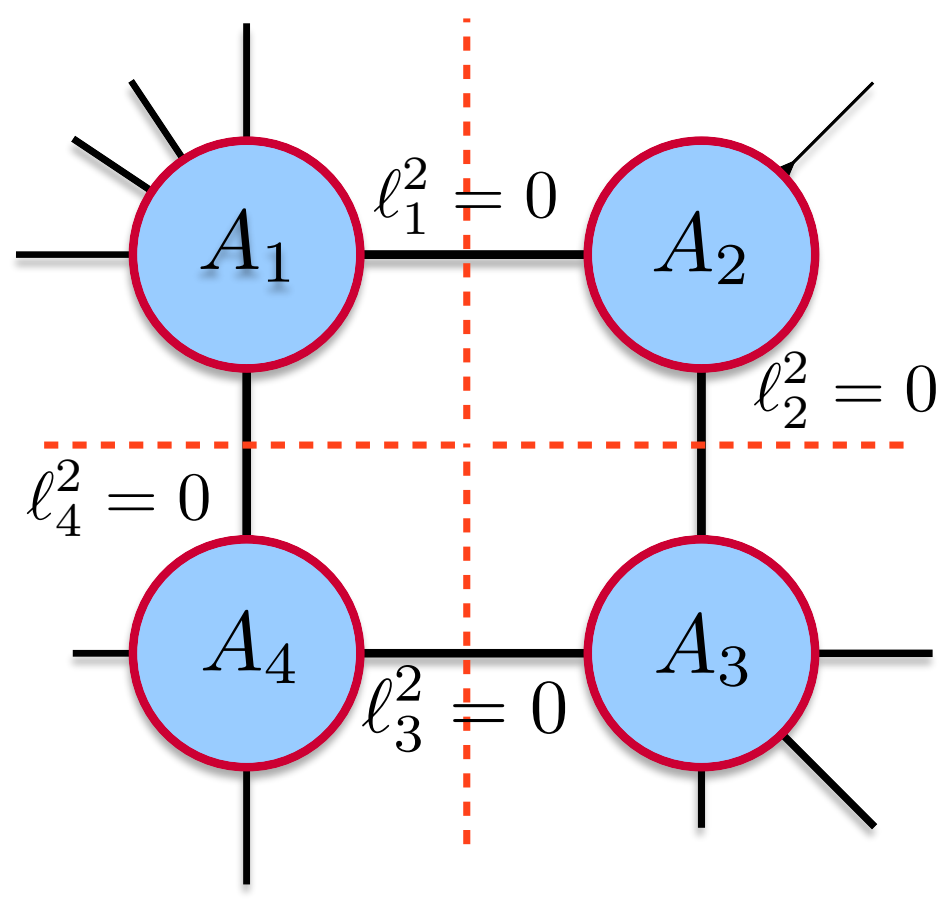

Figure 8: Factorizaton of a quadruple cut of a loop amplitude onto sub-amplitudes.

the modern methodology is a divide and conquer approach inspired by unitarity. The principles of unitarity have been around for a very long time and can be related back to the unitarity of the scattering matrix $S$. i.e. $S^{\dagger} S=1$ [90]. We normally write $S=1+i T$ where we isolate the forward scattering part. Now we can use the unitarity of $S$ to constrain $T$.

$$
\left(1-i T^{\dagger}\right)(1+i T)=1 \Longrightarrow i\left(T^{\dagger}-T\right)=T^{\dagger} T
$$

We identify $i\left(T^{\dagger}-T\right)$ as twice the imaginary part of $T$, which comes from its discontinuity across a branch cut.

We can use the analytic properties of the $S$ matrix, coupled with perturbation theory to address how these discontinuities play out at each order in perturbation theory. Assuming our scattering has a leading power of $g^{2}$ we have at NLO

$$
T=g^{2} T^{(0)}+g^{4} T^{(1)}+\ldots
$$

and putting into our equation we see that

$$
i g^{2}\left[\left(T^{(0) \dagger}-T^{(0)}\right)+g^{2}\left(T^{(1) \dagger}-T^{(1)}\right)\right]+\cdots=g^{4} T^{(0) \dagger} T^{(0)}+\ldots
$$


That is we see that

$$
\begin{aligned}
& \operatorname{Disc} T^{(0)}=0 \\
& \operatorname{Disc} T^{(1)}=T^{(0) \dagger} T^{(0)}
\end{aligned}
$$

The first line here simply re-affirms that tree-level scattering amplitudes are rational functions of the external kinematics. The second line tells us something interesting. We can reconstruct the discontinuity of a one-loop amplitude across some particular cut from the product of two tree level amplitudes. Implicit in the above equation is that we integrate over the final state particles connecting the two $T$ matrices together. We also implicitly sum over the helicities and types of particle which can propagate inside of the cut.

Lets consider a specific discontinuity associated with a grouping of external momentum, lets say $s_{12}$ where there are $n$ final state particles present. What the above equation is telling us is that we can compute the discontinuity of the one-loop associated with $s_{12}=\left(p_{1}+p_{2}\right)^{2}$ as follows

$$
\operatorname{Disc}\left(s_{12}\right)=(2 \pi)^{2} \sum_{h} \int \frac{d^{d} \ell}{(2 \pi)^{d}} \delta^{(+)}(\ell) \delta^{(+)}\left(\ell_{2}\right) A_{4}^{(0)}\left(\ell^{h_{1}}, 1,2,-\ell_{2}^{h_{2}}\right) A_{n}^{(0)}\left(-\ell^{-h_{1}}, 3, \ldots, n, \ell_{2}^{-h_{2}}\right)(3.8)
$$

where $\ell_{2}=\ell-P_{12}$ and $\delta^{(+)}(p)=\Theta\left(p^{0}\right) \delta\left(p^{2}\right)$ puts the cut momentum on their mass-shell with real positive energy solutions. The remaining integration corresponds to the integration over the two-body final state phase space of the cut particles.

The power of the unitarity method comes when we couple it with a Feynman diagrammatic approach. That is to say we couple it with our basis expansion given in equation 3.1. As stated previously the coefficients of our basis are rational functions in the external kinematics, therefore in order to have a discontinuity associated with $s_{12}$ the basis integral $I_{j}$ must depend on $s_{12}$ as one arguments. That is to say the set of propagators $\ell^{2}$ and $\left(\ell-P_{12}\right)^{2}$ must be present in the loop. We therefore write

$$
\operatorname{Disc}\left(s_{12}\right)=\sum_{i \in i_{12}} d_{i} \operatorname{Disc}\left(I_{4}^{i}\right)+\sum_{j \in j_{12}} c_{j} \operatorname{Disc}\left(I_{3}^{i}\right)+b_{12} \operatorname{Disc}\left(I_{2}^{s_{12}}\right)
$$

In the above equation $i_{12}$ denotes the set of all box integrals which contain $\ell-P_{12}$ as a propagator (and similarly $j_{12}$ for the triangles), since there are multiple box configurations which have this propagator we sum over all of these terms. There is of course, only one bubble which enters the sum, since there is only one bubble which has the correct pairing of propagators. We note that there is no process specific information in each $\operatorname{Disc}\left(I_{n}^{i}\right)$ since they are scalar integrals these can be computed once and for all. So by equating eqs. 3.8 and 3.9 we obtain information about the unknown coefficients $d, c$ and $b$ which enter into our calculation. This is the heart of the unitarity method pioneered by Bern, Dixon, Dunbar and Kosower in the mid 90's [91, 92] and was used to compute previously intractable problems [93, 94, 78].

\subsubsection{Generalized unitarity and quadruple cuts}

A drawback of the method described above is the entanglement of the various coefficients with one-another. As we perform cuts across different groupings of final state momenta we invariably 
encounter multiple boxes and triangles which enter into each of our cuts. Separating out the various contributions can be a time consuming and tricky task. We can significantly enhance the unitarity method if we drop one of the requirements, namely that our cut momentum correspond to physical momentum which have real positive definite momentum. Instead we think about a set of solutions to the loop momentum which solve the constraint equations $\ell_{1}^{2}=\ell_{2}^{2}=0$ where $\ell_{1}$ and $\ell_{2}$ are two of our loop propagators entering into our Feynman diagram. By summing all of the Feynman diagrams under this solution we obtain the following

$$
\frac{1}{\ell_{1}^{2}} A_{1}^{(0)}\left(\ell_{1},\left\{P_{1}\right\},-\ell_{2}\right) \frac{1}{\ell_{2}^{2}} A_{2}^{(0)}\left(\ell_{2},\left\{P_{2}\right\},-\ell_{1}\right)
$$

Where $A_{1}^{(0)}$ and $A_{2}^{(0)}$ denote tree-level amplitudes which depend on our cut momentum $\ell_{1}$ and $\ell_{2}$ and subsets of the external momenta $\left\{P_{1}\right\}$ and $\left\{P_{2}\right\}$. Our usual result for the calculation of the discontinuity is obtained by using the Cutkosky rules to replace the propagators by delta functions as follows

$$
\frac{1}{\ell^{2}} \rightarrow(2 \pi) \delta^{(+)}(\ell)
$$

and we recover the integrand from the previous section.

In principle there is no reason to stop at two constraints. If we assume that the loop momentum is four dimensional then we can impose up to four constraints on our loop momentum [95], i.e. we choose four pairings of the loop momentum and external kinematics

$$
\ell_{1}=\ell \quad \ell_{2}=\ell-P_{1} \quad \ell_{3}=\ell-P_{4} \quad \ell_{4}=\ell-P_{3}
$$

and demand that $\ell_{1}^{2}=\ell_{2}^{2}=\ell_{3}^{2}=\ell_{4}^{2}$. For this configuration our one-loop integrand will factor onto the product of 4 tree-level amplitudes

$$
\frac{1}{\ell_{1}^{2} \ell_{2}^{2} \ell_{3}^{2} \ell_{4}^{2}} A_{1}^{(0)}\left(\ell_{1}, \ell_{4}\right) A_{2}^{(0)}\left(\ell_{1}, \ell_{2}\right) A_{3}^{(0)}\left(\ell_{2}, \ell_{3}\right) A_{4}^{(0)}\left(\ell_{3}, \ell_{4}\right)
$$

Since there are four degrees of freedom for $\ell$ and four constraints we completely solve for the loop momentum algebraically and we can write

$$
A_{1}^{(0)}\left(\ell_{1}, \ell_{4}\right) A_{2}^{(0)}\left(\ell_{1}, \ell_{2}\right) A_{3}^{(0)}\left(\ell_{2}, \ell_{3}\right) A_{4}^{(0)}\left(\ell_{3}, \ell_{4}\right)=d_{i}
$$

Whats left is, upon application of the Cutkosky rules, the cut of a Feynman integral with four propagators, there is only one term in our basis expansion which has this, namely the scalar box associated with our specific choice of external momentum $I_{4}^{P_{1}, P_{2}, P_{3}}$. Therefore we have, using four cuts instead of two, uniquely isolated a single box coefficient. By doing every allowed four cut we can then identify every box coefficient which enters into our one-loop expansion.

With the boxes in hand we can then turn our attention to three-cuts, fixing three out of the four loop momentum, and leaving the cut integral as a single parameter integral. Now the boxes will pollute our triple cut and we will be left with a single triangle, and multiple boxes, but by a clever parametrization of our solution we can easily disentangle the two types of contribution and isolate the single unknown triangle coefficient. Similarly with the double cut we parameterize our loop 
momentum solution such that the extraction of the unknown bubble coefficient is also simple. By this repeated divide and conquer approach the individual coefficients in the basis can be isolated. There are complications and subtleties with each method, such as in order to fully reconstruct the amplitude some $d$ dimensional information must be restored to isolate the rational term. But by and large this repeated cutting and multiple particle factorization is what lies at the heart of automatic one-loop generators. Its probably not the best use of our time together for me to go into explicit detail about each method, as I'd also like to cover sufficient material on IR regularization. So instead I'll give a quick box example, as they are analytic problems. Afterwards you'll be in a good position to learn more about the methods if you are interested. I wholeheartedly recommend the following references $[95,96,97,98,99,100,101,102]$ if you wish to learn more.

3.2.3 Example : $e^{+} e^{-} \rightarrow q \bar{q} g$.

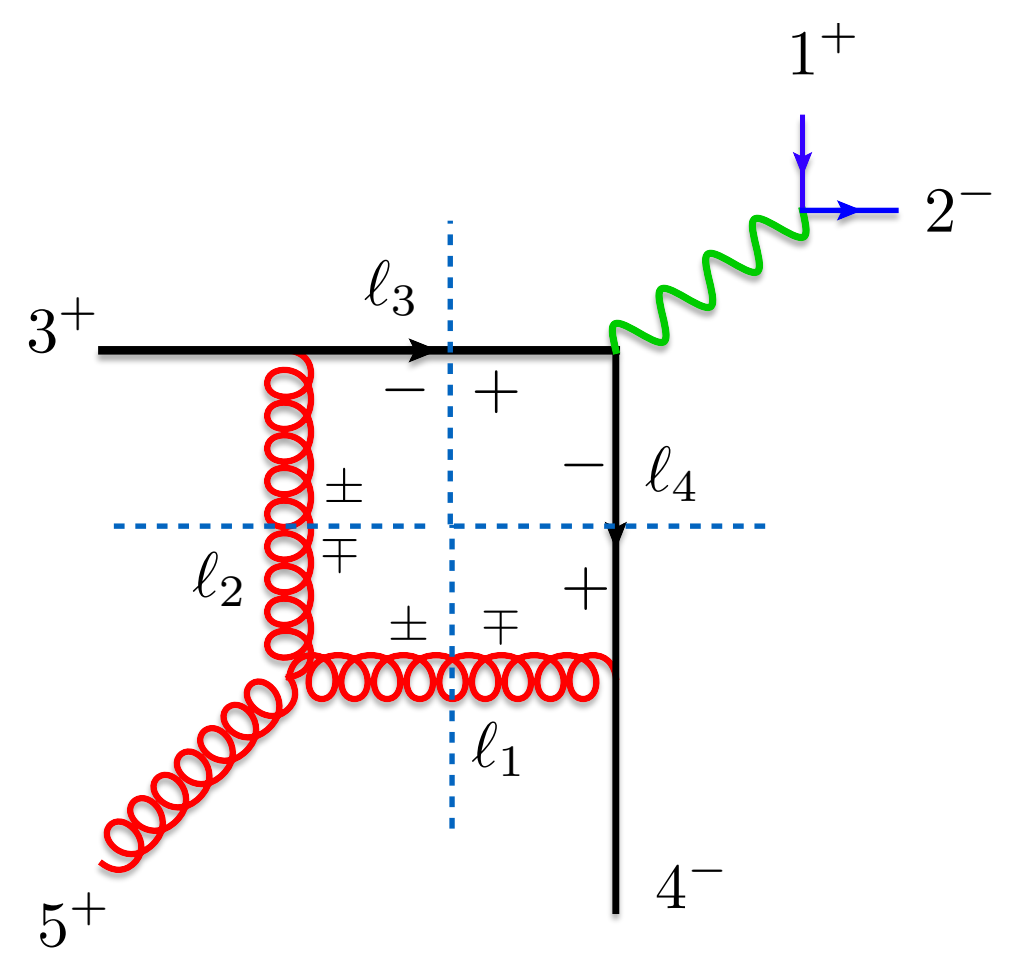

Figure 9: An example of a one-bass box coefficient for the Leading Color contribution for $e^{+} e^{-} \rightarrow q \bar{q} g$

As an example lets consider the box coefficient which occurs in the process $e^{+} e^{-} \rightarrow q \bar{q} g$ at one-loop. We'll look at the leading color contribution, which is sketched out in Fig 9. There is a single box which has the leptons as a massive corner and three massless legs (we call it a onemass box). The coefficient of the box is obtained from the product of four tree-amplitudes, the cuts are illustrated in the figure. The helicities of the fermion line is completely fixed by helicity conservation at each vertex (recall that we have an all outgoing convention so the particles flip helicity across a cut line). And we need at least one negative helicity gluon at the triple gluon vertex to obtain a non-zero amplitude. The helicity of the internal gluon propagators therefore 
appears to take two possible assignments, which are explicitly labelled in Fig. 10. We note that one has an alternating MHV and $\overline{\mathrm{MHV}}$ structure and one does not. A consequence of complex momentum solutions to the three-point amplitude is that the configuration which does not have the alternating structure vanishes. This can be readily seen. We require that $\left|\ell_{1}\right\rangle \propto|4\rangle$ from the bottom right vertex to ensure the solution to the cut condition coincides with a non-vanishing three point amplitude (which depends on $\left.\mid \ell_{1}\right]$ ). However a similar requirement from the adjacent vertex also imposes that $\left|\ell_{1}\right\rangle \propto|5\rangle$. This can only be satisfied if the unphysical restraint $p_{4} \propto p_{5}$ is true. Therefore this contribution must vanish. Our remaining option gives,
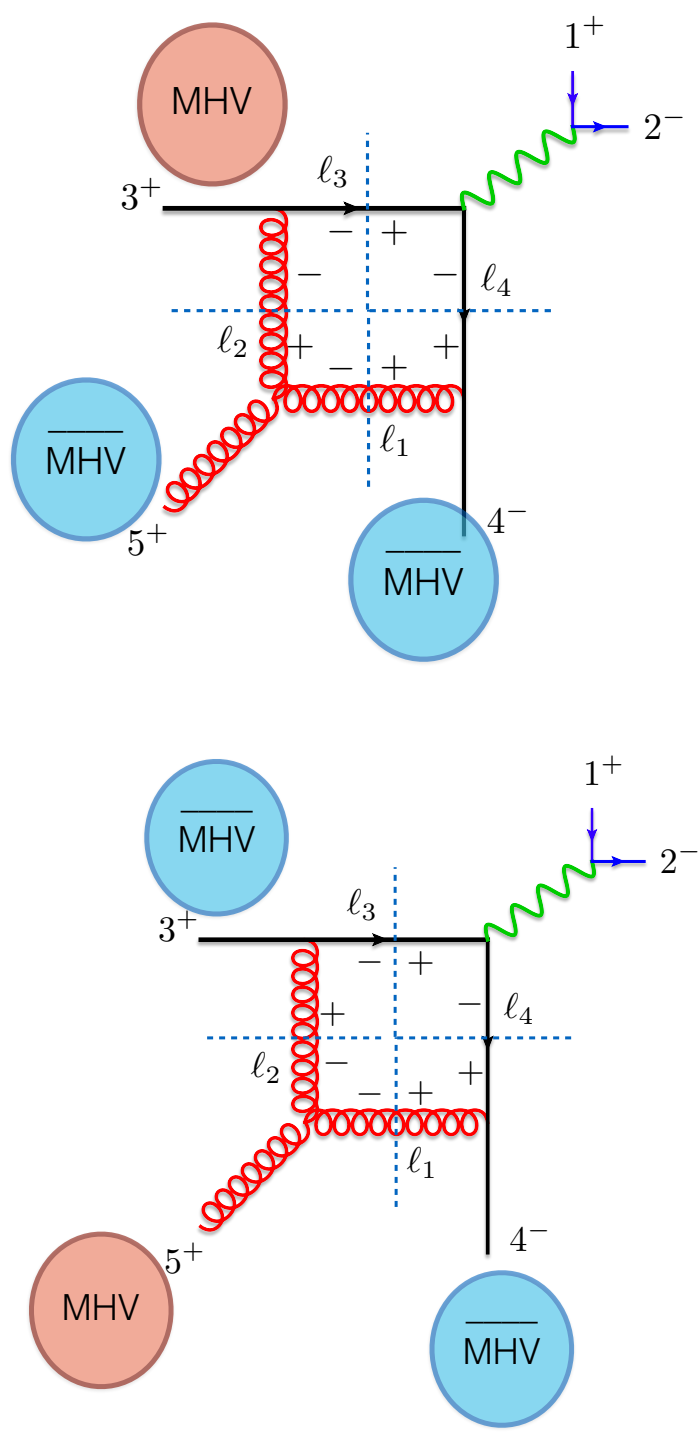

Figure 10: Possible assignments of helicity for our quadruple cut example for $e^{+} e^{-} \rightarrow q \bar{q} g$

$A_{5}^{(1)}\left(1_{\bar{e}}^{+}, 2_{e}^{-}, 3_{q}^{+}, 4_{\bar{q}}^{-}, 5_{g}^{+}\right)_{d_{1}}=A_{3}^{g g g}\left(\ell_{1}^{-}, 5^{+}, \ell_{2}^{-}\right) A_{3}^{g q \bar{q}}\left(\ell_{2}^{+}, 3^{+}, \ell_{3}^{-}\right) A_{4}^{q \ell^{+} \ell^{-} \bar{q}}\left(\ell_{3}^{+}, 1^{+}, 2^{-}, \ell_{4}^{-}\right) A_{3}^{q \bar{q} g}\left(\ell_{4}^{+}, 4^{-},\left(B_{\mathrm{r}}^{+} 1\right) 5\right)$ 
Now we have to setup the solutions for the loop momentum $\ell_{i}$, which means that each of the four loop momenta is on-shell $\ell_{i}^{2}=0$. Momentum conservation defines each constraint equation as

$$
\begin{aligned}
& \ell_{2}^{2}=0 \\
& \ell_{3}^{2}=\left(\ell_{2}-p_{3}\right)^{2}=0 \\
& \ell_{4}^{2}=\left(\ell_{2}-P_{123}\right)^{2}=0 \\
& \ell_{1}^{2}=\left(\ell_{2}+p_{5}\right)^{2}=0
\end{aligned}
$$

We have four constraint equations and four-unknowns, so we can completely solve $\ell_{2}$. We begin by choosing our parameterization of $\ell_{2}$ which can be written in-terms of a basis of four mutually orthogonal light-like momenta

$$
\ell_{2}^{\mu}=\alpha p_{3}^{\mu}+\beta p_{5}^{\mu}+\frac{\delta}{2}\left\langle 3\left|\gamma^{\mu}\right| 5\right]+\frac{\rho}{2}\left\langle 5\left|\gamma^{\mu}\right| 3\right]
$$

We'll now use the constraint equation to solve for the coefficients, firstly we see that the second condition implies that $p_{3} \cdot \ell_{2}=0$ which tells us that

$$
0=\beta p_{3} \cdot p_{5}
$$

and $\beta=0$. Similarly the final constraint implies that $\alpha=0$. The on-shell condition implies the following

$$
0=\frac{\delta \rho}{2} s_{35}
$$

So either $\delta$ or $\rho$ are zero. There are thus two solutions which satisfy the propagator equations (as they're quadratic this is hardly a surprise). We take $\rho=0$ so that

$$
\ell_{2}^{\mu}=\frac{\delta}{2}\left\langle 3\left|\gamma^{\mu}\right| 5\right]
$$

The final condition allows us to solve for $\delta$ since $2 \ell_{2} \cdot P_{123}=s_{123}$ or more conveniently $-2 \ell_{2} \cdot P_{45}=$ $s_{45}$ and

$$
-\delta\langle 3|4| 5]=s_{45} \Longrightarrow \delta=\frac{\langle 54\rangle}{\langle 34\rangle}
$$

We can thus write the loop momentum as follows

$$
\ell_{2}^{\mu}=\frac{\langle 54\rangle}{2\langle 34\rangle}\left\langle 3\left|\gamma^{\mu}\right| 5\right]
$$

Using the following gluon 3-point MHV amplitude

$$
A_{3}^{g g g}\left(1^{-}, 2^{+}, 3^{-}\right)=\frac{\langle 13\rangle^{3}}{\langle 12\rangle\langle 23\rangle}
$$

We can construct our coefficient as follows, the four tree amplitudes give us the following

$$
d_{1}=\left(\frac{\left\langle\ell_{1} \ell_{2}\right\rangle^{3}}{\left\langle\ell_{1} 5\right\rangle\left\langle\ell_{2} 5\right\rangle}\right)\left(-\frac{\left[3 \ell_{2}\right]^{2}}{\left[3 \ell_{3}\right]}\right)\left(\frac{\left\langle\ell_{4} 2\right\rangle^{2}}{\left\langle\ell_{3} \ell_{4}\right\rangle\langle 12\rangle}\right)\left(\frac{\left[\ell_{4} \ell_{1}\right]^{2}}{\left[4 \ell_{4}\right]}\right)
$$


We now proceed to systematically remove $\ell_{1}$ and $\ell_{3}$ and $\ell_{4}$ using momentum conservation, for example

$$
\left\langle\ell_{4} 2\right\rangle^{2}\left[\ell_{4} \ell_{1}\right]^{2} \rightarrow\left\langle 2\left|\ell_{4}\right| \ell_{1}\right]^{2}=\left\langle 2\left|\ell_{2}+P_{45}\right| \ell_{1}\right]^{2}
$$

Repeated application of this type of identity allows us to re-write the coefficient solely in terms of $\ell_{2}$

$$
d_{1}=\frac{\left[3 \ell_{2}\right][52]\left\langle 2\left|\ell_{2}+4\right| 5\right]^{2}}{\langle 12\rangle\left[2 \ell_{2}\right][54]}
$$

At this stage we can already see why we choose the $\rho=0$ option for our loop momentum. The $\delta=$ 0 solution would have given as a solution where $\left.\left.\mid \ell_{2}\right] \propto \mid 3\right]$, and as a result this solution would vanish for this particular example (for simple one box topologies like this one this is not uncommon).

We can finish our calculation by making our replacement for our loop momentum solution finding,

$$
d_{1}=-\frac{\langle 24\rangle^{2}[53][54]}{\langle 12\rangle}=s_{35} s_{45} A_{5}^{(0)}\left(1_{\bar{e}}^{+}, 2_{e}^{-}, 3_{q}^{+}, 4_{\bar{q}}^{-}, 5_{g}^{+}\right)
$$

That is the box coefficient factorizes nicely onto the tree-level result.

\subsection{Real Corrections}

The previous section discussed virtual corrections. There is a second source of higher order corrections, corresponding to when an additional particles are radiated from the tree-level topology. Since these particles can (in principle) be observed in the final state, these corrections are known as "real" corrections. On the one hand real corrections are simpler to compute, since they have less intricate mathematical structure then virtual corrections. However they also introduce their own complexities when attempting to make Monte Carlo code to perform phenomenology at the LHC. This is because, while the extra emission can in be observed in the detector as hard radiation, it is not required to be there (since the requirements define the LO final state). This means that we include regions of phase space in which additional emission are associated with vanishing (or unresolved) emissions. In these regions of phase space the matrix element becomes singular, as it must, since these regions of phase space exactly compensate the IR singularities arising from the virtual matrix elements. Thankfully the singular behavior of tree-level matrix elements can be understood via factorization. In dealing with NLO computations this is critical, since it allows us to apply universal terms which can be used in any QCD computation. We'll now study the different limits of QCD amplitudes to get a feeling for the factorization

\subsubsection{Soft limits of scattering amplitudes}

We recall our amplitude for $e^{+} e^{-} \rightarrow q \bar{q} g$.

$$
A_{5}^{(0)}\left(1_{\bar{e}}^{+}, 2_{e}^{-}, 3_{q}^{+}, 4_{\bar{q}}^{-}, 5_{g}^{+}\right)=\frac{\langle 34\rangle}{\langle 35\rangle\langle 54\rangle}\left(\frac{\langle 24\rangle^{2}}{\langle 12\rangle\langle 34\rangle}\right)
$$


What happens to the amplitude as $p_{5} \rightarrow 0$ ? Well the above factorization is already manifest we can write

$$
A_{5}^{(0)}\left(1_{\bar{e}}^{+}, 2_{e}^{-}, 3_{q}^{+}, 4_{\bar{q}}^{-}, 5_{g}^{+}\right)=\mathscr{S}\left(3,4,5^{+}\right) \times A_{4}^{(0)}\left(1_{\bar{e}}^{+}, 2_{e}^{-}, 3_{q}^{+}, 4_{\bar{q}}^{-}\right)
$$

where we have defined the following Soft (or Eikonal) factor

$$
\mathscr{S}\left(a, b, c^{+}\right)=\frac{\langle a b\rangle}{\langle a s\rangle\langle s b\rangle} \quad \mathscr{S}\left(a, b, c^{-}\right)=-\frac{[a b]}{[a s][s b]}
$$

In the above notation $c$ is the soft gluon and $a$ and $b$ are the two adjacent colored particles. Although this was a very simple example this is actually representative of the general factorization of QCD amplitudes in the limit of a soft gluon emission

$$
A_{n}^{(0)}\left(1,2, \ldots, a, s^{+/-}, b, \ldots n\right) \rightarrow_{p_{s} \rightarrow 0} \mathscr{S}\left(a, b, s^{ \pm}\right) A_{n-1}^{(0)}(1,2, \ldots, a, b, \ldots, n)
$$

You may worry that we only proved this for gluons going soft with respect to a quark pair. But this is is fact independent of wether the adjacent particles are quarks or gluons. The soft limit of QCD is insensitive to the emitters helicity, or spin, it only depends the angular direction of the emitting particles.

\subsubsection{Collinear limits}

The other type of IR singularity occurs when two particles become collinear (or parallel) to one another. In our example $e^{+} e^{-} \rightarrow q \bar{q} g$, such singular configurations would correspond to the gluon becoming collinear to either one of the fermions. In the collinear limit (e.g. 3\|5) we see that

$$
s_{35}=\left(p_{3}+p_{5}\right)^{2} \rightarrow\left(p_{3}+\lambda p_{3}\right)^{2}=(1+\lambda)^{2} p_{3}^{2}=0
$$

So the invariant $s_{a b}$ associated with the parallel particles $a \| b$ vanishes. Normally we do not pick out $a$ or $b$ to be special (as done in the formula above), but instead introduce a new momentum $p_{c}=p_{a}+p_{b}$ which both are parallel to i.e.

$$
p_{a} \approx z p_{c} \quad p_{b} \approx(1-z) p_{c}
$$

such that overall momentum is conserved in the limit. We can use the above equations to relate how the spinors behave in the limit,

$$
\begin{aligned}
|a\rangle \approx \sqrt{z}|c\rangle & \mid a] \approx \sqrt{z} \mid c] \\
|b\rangle \approx \sqrt{1-z}|c\rangle & \mid b] \approx \sqrt{1-z} \mid c]
\end{aligned}
$$

Taking this limit in our amplitude (3\||5) we see that

$$
\begin{aligned}
A_{5}^{(0)}\left(1_{\bar{e}}^{+}, 2_{e}^{-}, 3_{q}^{+}, 4_{\bar{q}}^{-}, 5_{g}^{+}\right) & =\frac{\langle 34\rangle}{\langle 35\rangle\langle 54\rangle}\left(\frac{\langle 24\rangle^{2}}{\langle 12\rangle\langle 34\rangle}\right) \\
& \approx \frac{1}{\sqrt{1-z}\langle 35\rangle} \frac{\langle 24\rangle^{2}}{\langle 12\rangle\langle 4 c\rangle} \\
& =\text { Split_}_{-}\left(3_{q}^{+}, 5_{g}^{+} ; z\right) A_{4}^{(0)}\left(1_{\bar{e}}^{+}, 2_{e}^{-}, c_{q}^{+}, 4_{\bar{q}}^{-}\right)
\end{aligned}
$$


Where we have introduced the splitting amplitude Split_ $\left(3_{q}^{+}, 5_{g}^{+} ; z\right)$. This is a specific example of the general factorization of QCD amplitudes in this limit

$$
A_{n}^{(0)}\left(1,2, \ldots, a^{\lambda_{a}}, b^{\lambda_{b}}, \ldots n\right) \rightarrow_{a \| b} \sum_{\lambda_{c}= \pm} \operatorname{Split}_{-}\left(a^{\lambda_{a}}, b^{\lambda_{b}} ; z\right) A_{n-1}^{(0)}\left(1,2, \ldots, c^{\lambda_{c}}, \ldots, n\right)
$$

Note the differences with respect to the soft limit. Here the species and spin of the particles involved in the limit is crucial. In general we have to sum over both possible helicity states of the combined particle $c$. However in our example there is only one non-zero contribution.

\section{Dealing with the IR singularities}

Next we would like to deal with the IR singularities, so that we can construct a working Monte Carlo code. We've seen the basic problem over the last couple of sections.

- Loop integrals contain divergences which manifest themselves as poles in $1 / \varepsilon$ (virtual)

- Real emission amplitudes are singular in regions of phase space where emitted partons can be soft or collinear.

These two types of singularities are intimately related, but at first glance appear rather different. The virtual singularities reside in the LO phase space (i.e. they have the same number of final state particles as the LO process). The real singularities have an extra emission which must be integrated over inclusively and thus have a larger phase space for integration. To get the idea of how to proceed its actually easiest to introduce a toy model which shares the same spirit as the full QCD calculation, but has simpler mathematical structure. I've taken this toy model from the one presented in the Black Book of QCD by John Campbell, Joey Huston and Frank Krauss [59]. This book is an excellent comprehensive overview of QCD and I thoroughly recommend it to you.

Our toy model is constructed as follows, we first consider a LO (Born) matrix element (with $n$ particles) which is IR and UV finite (we can treat it as a constant for now)

$$
\mathscr{B}_{n}=\sum\left|\mathscr{M}_{n}^{(0)}\right|^{2}
$$

We now compute NLO corrections in our toy model, obtaining virtual and real corrections. We assume that any UV divergences in our model have been removed and that the remaining renormalized virtual correction is of the form

$$
\mathscr{V}_{n}=\frac{V_{n}}{\varepsilon}=\sum\left|\mathscr{M}_{n}^{(0)} \mathscr{M}_{n}^{*(1)}\right|
$$

That is a constant divided by a single pole in $\varepsilon$. Finally in our toy example the real emission matrix depends only on a one-dimensional phase-space parameter $x \in[0,1]$ and the matrix element diverges as $x \rightarrow 0$. So that we can write

$$
\mathscr{R}_{n}(x)=\frac{R_{n}(x)}{x}
$$

Where $R_{n}$ is a regular function over the full integral. 
Our NLO cross section is thusly obtained by integrating out $x$ and combining it with the Born and Virtual contributions, i.e.

$$
\sigma^{N L O}=\left[\mathscr{B}_{n}+\mathscr{V}_{n}\right] F_{n}^{J}+\int_{0}^{1} d x \mathscr{R}_{n}(x) F_{n+1}^{J}(x)
$$

In the above equation I've introduced one new factor, the function $F_{n}^{J}$. This plays the role of defining an infrared-safe observable. We can think about this as ensuring that the LO process is free of singularities. For example if we were looking at 3-jet production at the LHC we would require the jets to be energetic enough ( above a certain $p_{T}$ ) and well-separated from each other to avoid overlapping phase space regions. The virtual part shares the same phase space as the Born, so the same function can be applied there too. We have to be a little bit more careful in the real contribution, since the presence of $x$ could alter the function. In order for our calculation to make sense it must be the case that

$$
\lim _{x \rightarrow 0} F_{n+1}^{J}(x)=F_{n}^{J}
$$

This criterion is our toy model manifestation of the need to work with infrared-safe observables. Otherwise the concept of NLO corrections loses meaning. Inserting our definitions of the toy model the problem we are trying to solve is

$$
\sigma^{N L O}=\left[\mathscr{B}_{n}+\frac{V_{n}}{\varepsilon}\right] F_{n}^{J}+\int_{0}^{1} \frac{d x}{x} R_{n}(x) F_{n+1}^{J}(x)
$$

We'll now look at some techniques for computing our NLO cross section.

\subsection{Phase space slicing}

We begin by implementing a regularization prescription on our divergent real integral. We modify the $1 / x$ term, replacing it with $x^{-1-\varepsilon}$ such that our desired physical amplitude (and associated divergence) occurs at $\varepsilon=0$. Our NLO correction is then given by

$$
\sigma^{(1)}=\frac{V_{n}}{\varepsilon} F_{n}^{J}+\int_{0}^{1} \frac{d x}{x^{1+\varepsilon}} R_{n}(x) F_{n+1}^{J}(x)
$$

We are free to introduce a parameter $\delta$ which lies somewhere in our integration range, splitting the integral accordingly.

$$
\sigma^{(1)}=\frac{V_{n}}{\varepsilon} F_{n}^{J}+\int_{0}^{\delta} \frac{d x}{x^{1+\varepsilon}} R_{n}(x) F_{n+1}^{J}(x)+\int_{\delta}^{1} \frac{d x}{x^{1+\varepsilon}} R_{n}(x) F_{n+1}^{J}(x)
$$

At the moment we've simply done some PR. However if we choose $\delta$ such that $\delta<<1$ then we can approximate

$$
R_{n}(x) F_{n+1}^{J}(x) \approx R_{n}(0) F_{n}^{J}
$$

in the first integral, yielding

$$
\sigma^{(1)}=\frac{V_{n}}{\varepsilon} F_{n}^{J}+R_{n}(0) F_{n}^{J} \int_{0}^{\delta} \frac{d x}{x^{1+\varepsilon}}+\int_{\delta}^{1} \frac{d x}{x^{1+\varepsilon}} R_{n}(x) F_{n+1}^{J}(x)
$$


Or, re-arranging

$$
\sigma^{(1)}=\left(\frac{V_{n}}{\varepsilon}+R_{n}(0) \int_{0}^{\delta} \frac{d x}{x^{1+\varepsilon}}\right) F_{n}^{J}+\int_{\delta}^{1} \frac{d x}{x^{1+\varepsilon}} R_{n}(x) F_{n+1}^{J}(x)
$$

The second integral is no longer divergent for any non-zero choice of $\delta$, so we can set $\varepsilon=0$ here. The integral in the second integral we can write

$$
\sigma^{(1)}=\left(\frac{V_{n}}{\varepsilon}-\frac{\delta^{-\varepsilon}}{\varepsilon} R_{n}(0)\right) F_{n}^{J}+\int_{\delta}^{1} \frac{d x}{x} R_{n}(x) F_{n+1}^{J}(x)
$$

Expanding as a power series in $\varepsilon$ we find.

$$
\sigma^{(1)}=\left(\frac{\left(V_{n}-R_{n}(0)\right)}{\varepsilon}+\log \delta R_{n}(0)\right) F_{n}^{J}+\int_{\delta}^{1} \frac{d x}{x} R_{n}(x) F_{n+1}^{J}(x)
$$

Our toy model will thus obey the KLN theorem if the following result holds

$$
\lim _{x \rightarrow 0} R_{n}(x)=R_{n}(0)=V_{n}
$$

Assuming this is the case then our final correction is given by

$$
\sigma^{(1)}=\log \delta \cdot V_{n} F_{n}^{J}+\int_{\delta}^{1} \frac{d x}{x} R_{n}(x) F_{n+1}^{J}(x)
$$

There are several things to say about this result. Firstly we note that the dependence on $\varepsilon$ is completely gone, we can set $\varepsilon=0$ everywhere with imputinty and integrate our results in fourdimensions. Secondly we recall the approximate nature of how we simplified the integration of the real matrix element in the region of small $\delta$. This is by nature an approximation, so we know that it will break with corrections expected at $\mathscr{O}(\delta)$. The method is therefore only useful for any kind of practical application if $\delta$ is taken to be very small, such that the power corrections not included in our approximation are negligible in the total. The drawback of this approach is immediately obvious from closer inspection of the residual integral over the finite phase space of the real amplitude. Since $\delta$ is now very small we push ourselves towards the divergent region of the real matrix element. This means that this integral is rather numerically unstable, and requires significant computational resources to perform (how significant depends on what sort of calculation we are doing). Further as $\delta$ shrinks the magnitude of both the virtual term and the real term grow logarithmically (in this toy model, they actually grow like $\log ^{2}$ at NLO in QCD and $\log ^{4}$ at NNLO). Large numerical cancellations such as these are very undesirable from a computational viewpoint, since this means that both have to be integrated to a very high precision. On the plus side however, this method is extremely easy to implement, especially if you have a good way of doing the resolved integral, since this always has non-zero emission it looks like a tree-level process. Finally there is the term $R_{n}(0) F_{n}^{J}$ itself, which is used in the approximation. This has to be constructed appropriately from the un-resolved limits of the real matrix element. We know the factorzation properties of QCD amplitudes, so there is some hope. But there are still complications which render this bit tricky, for example even at NLO the situation is not $100 \%$ obvious. Consider a process with three final state partons $p_{1}$ and $p_{2}$ and $p_{i}$, where we assume that $p_{1}$ and $p_{2}$ are hard fermions and $p_{i}$ is an emitted gluon which can be soft or collinear. If $s_{1 i} \rightarrow 0$ and $s_{i 2} \neq 0$ then 


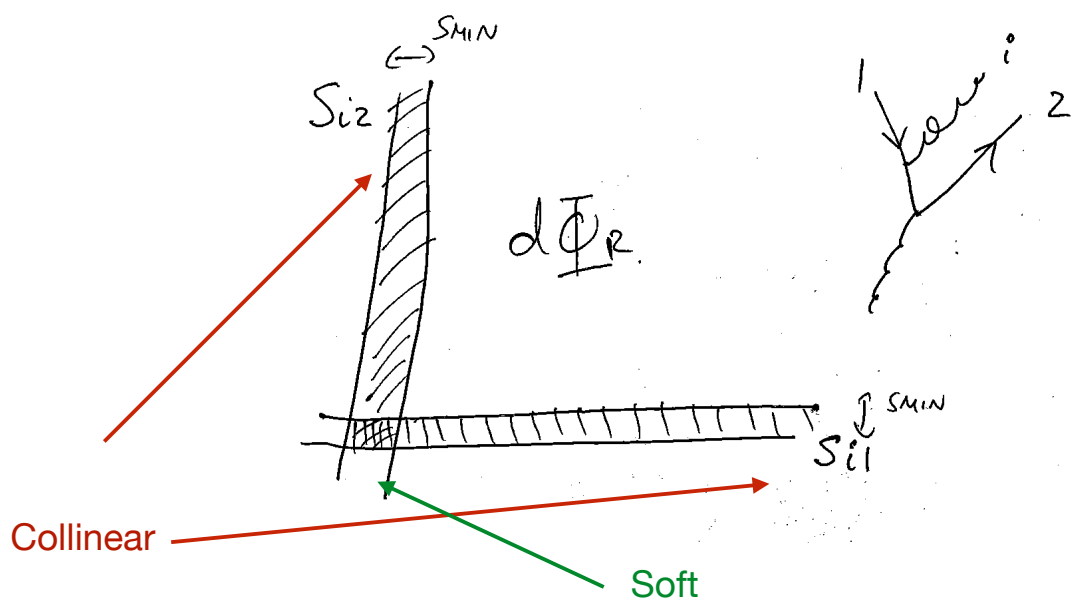

Figure 11: Phase space slicing at NLO

the gluon is collinear to $p_{1}$ and the approximation we would like to use for our matrix element in this limit is given by our splitting function multiplying the LO matrix element for 2 final state fermions. However what happens as $s_{i 2} \rightarrow 0$ ? Then we recover the soft limit for which we want to use our Eikonal approximation. If we simply included both we would double count, since this soft limit is present in the collinear limit too. We therefore have to sort out the ordering of the limits such that we can separate the collinear and soft limits accordingly. This is illustrated in Figure 11, along either one of the axis the collinear approximation can be used, whereas at the origin the soft approximation should be employed.

At NLO this is a manageable task and the singularities can be just about handled using the prescription outlined above (truncating the collinear near the origin to avoid overlap with the soft region) $[27,28]$. However when we go up to NNLO we will find a huge increase in complexity, because now we can have two unresolved partons. This means that we get many different types of singularities which overlap with one another. Dealing with these singularities in a clever way to obtain an approximate matrix element in the unresolved region is an extremely non-trivial task.

\section{2 $N$-jettiness slicing at NNLO}

For the reasons discussed above phase space slicing fell out of favor over the last 20 years in favor of subtraction, which we'll discuss shortly. However recently there has been something of a renaissance of these methods at NNLO [24, 25] due to some clever factorization theorems for the below cut bit. One recent development is the idea of using the event shape variable $N$-jettiness [29] to classify the above and below cut contributions. Therefore in order to discuss this method I first have to introduce the $N$-jettiness event shape variable.

As a concrete example lets consider the production of a 1-jet final state at NNLO. We have various possible partonic configurations that can contribute at this order. Firstly we have LO phase space in which there are three partons (+ whatever EW stuff we made). Secondly we have a phase 


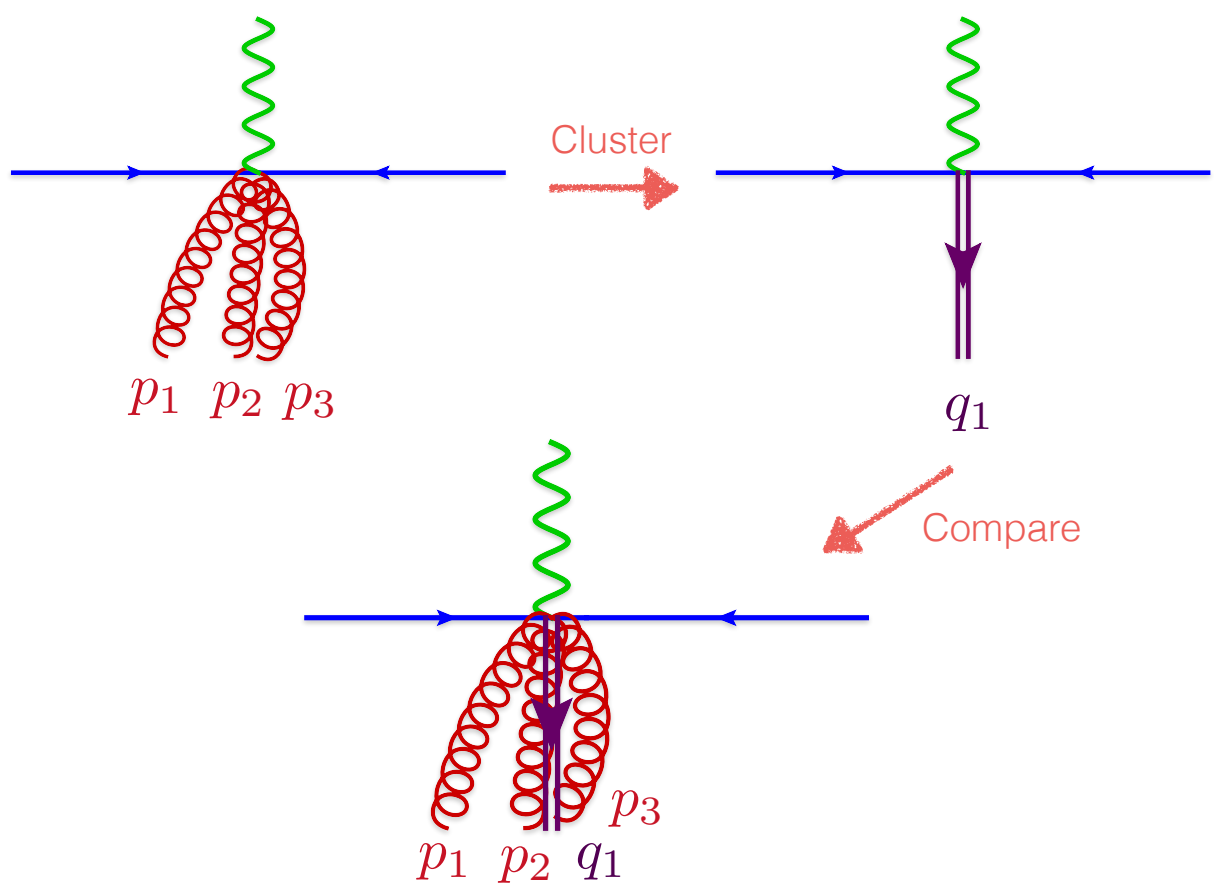

Figure 12: A representation of an event with a small value of $\mathscr{T}_{1}$, all three final state partonic momenta reside close to the jet-axis defined $q_{1}$ resulting in a small value of $\mathrm{f} \mathscr{T}_{1}$ (and double unresolved limits).

space with four partons (corresponding for instance to the mixed real-virtual corrections), finally we have the option of making 5 partons in the final state (which comes from the real-real corrections in which two tree-level amplitudes interfere with one-another). Lets focus on the double-real phase space as its the most complicated. Now we are interested in the jet-observable so we have to cluster our momentum using our favorite infra-red safe jet algorithm. After this we have 3 possibilities corresponding to:

1. all three partons are clustered into the same jet.

2. two partons cluster into one jet and another parton does not (this last parton could make a second jet if hard enough, but there is no mandate for it to do so)

3. all three partons are separated and no partons cluster together.

Now we think about the potential IR problems in each region. In region 1) we have lots of IR problems, there are many possible double unresolved limits here corresponding to collinear (for example all three partons travel in the same direction), and double soft limits etc. Region 2) has less types of singularities since the only truly nasty one is the double soft, or one soft and one collinear. Finally in region 3 no collinear singularities are possible, so we just worry about soft limits.

We want some way of knowing when these different regions develop problems, and it is here that the $N$-jettiness observable comes into play. For example, consider region 3), if all three partons 
are hard enough to form jets, then soft singularities are forbidden. If two jets are present we can have one soft parton, but not two. Finally when there is only one-jet we can have two soft partons. So clearly how " 1 -jet" like the event is can be utilized to discriminate between the sort of singularities present.

We need a more quantitive version of this statement, so we introduce the $N$-jettiness event shape [29]. We take our set of $M$ final state partonic (unclustered) momentum $\left\{p_{k}\right\}$ and our clustered jets $q_{i}$. Now we want to compute how 1-jetty the event is, so we take the hardest jet direction after clustering. The 1 -jettiness for a single parton $p_{j}$ is given by

$$
\mathscr{T}_{1}\left(p_{j}\right)=\min _{i=a, b, 1}\left(\frac{q_{i} \cdot p_{j}}{Q_{i}}\right)
$$

where $a$ and $b$ correspond to the initial state partons at the LHC. $Q_{i}$ is an energy scale associated with the jet which we typically take to be $Q_{i}=2 E_{i}$. The total jettiness of the event is given by the sum over the $M$ partons

$$
\mathscr{T}_{1}=\sum_{k=1}^{M} \mathscr{T}_{1}\left(p_{k}\right)=\sum_{k=1}^{M} \min _{i=a, b, 1}\left(\frac{q_{i} \cdot p_{k}}{Q_{i}}\right)
$$

Our claim is that $\mathscr{T}_{1}=0$ corresponds to the double unresolved limit of our double-real phase space. To see this note that the LO phase space has $q_{1}=p_{1}$ so $\mathscr{T}_{1}=0$. Now consider the configuration at NNLO shown in Figure 4.2. Three partons cluster together to form a single jet, since $q_{1}$ overlaps closely with $p_{1}+p_{2}+p_{3}$ all of the individual products entering our sum are small. As we approach a double unresolved limit $\mathscr{T}_{1} \rightarrow 0$, for example consider a triple collinear limit in which all three partons become parallel to $q_{1}$ and $\mathscr{T}_{1} \rightarrow 0$. Now consider the second example, illustrated in Fig. 13, in which we have a partonic emission $p_{3}$ which is hard enough to put us away from the soft limit, $q_{1}$ is formed out of $p_{1}$ and $p_{2}$ here. Now there is potential for $q_{1} \cdot p_{1}$ or $q_{1} \cdot p_{2}$ to go to zero, but not $q_{1} \cdot p_{3}$ (by construction since we assumed that $p_{3}$ was hard enough). The only possibility which causes us concern is a limit in which $p_{3}$ becomes soft, but not clustered. Here though we recover $\mathscr{T}_{1} \rightarrow 0$ as expected. So we see that $\mathscr{T}_{1}=0$ contains all of the double-unresolved limits, and $\mathscr{T}_{1}>0$ can have at most a single unresolved limit. The $\mathscr{T}_{1}>0$ region thus corresponds to the integration over non-zero $\delta$ in our toy model, and can be treated as a regular NLO calculation.

We still need to form a suitable approximation for $\mathscr{T}_{1} \approx 0$ so that we can use our slicing procedure. It is here that the magic of SCET comes into play. I dont have time to introduce SoftCollinear-Effective field Theory [33, 34, 35, 36, 37] in these lectures, but we can use two of the main features of the theory to help us here. 1) It nicely separates the soft and collinear regions using the tools of EFT (see your EFT lectures). 2) It provides a factorization theorem which can be employed at low $\mathscr{T}_{N}$. The factorziation theorem states that for small values of $\mathscr{T}_{N}$ less than some cut $\mathscr{T}_{N}^{\text {cut }}$. We can write the cross section as a convolution over the following terms

$$
\sigma\left(\mathscr{T}_{N}<\mathscr{T}_{N}^{\text {cut }}\right)=\int_{0}^{\mathscr{T}_{N}^{\text {cut }}} d \mathscr{T}_{N} B_{a} \otimes B_{b} \otimes S \otimes H \otimes \prod_{i=1}^{N} J_{i}+\mathscr{O}\left(\mathscr{T}_{N}^{\text {cut }}\right)
$$

Here $B_{i}$ are the beam functions [32], which describe collinear initial state radiation. $S$ is a soft function $[103,104]$ which describes soft radiation and $J_{i}$ are jet functions $[105,106]$ that model 

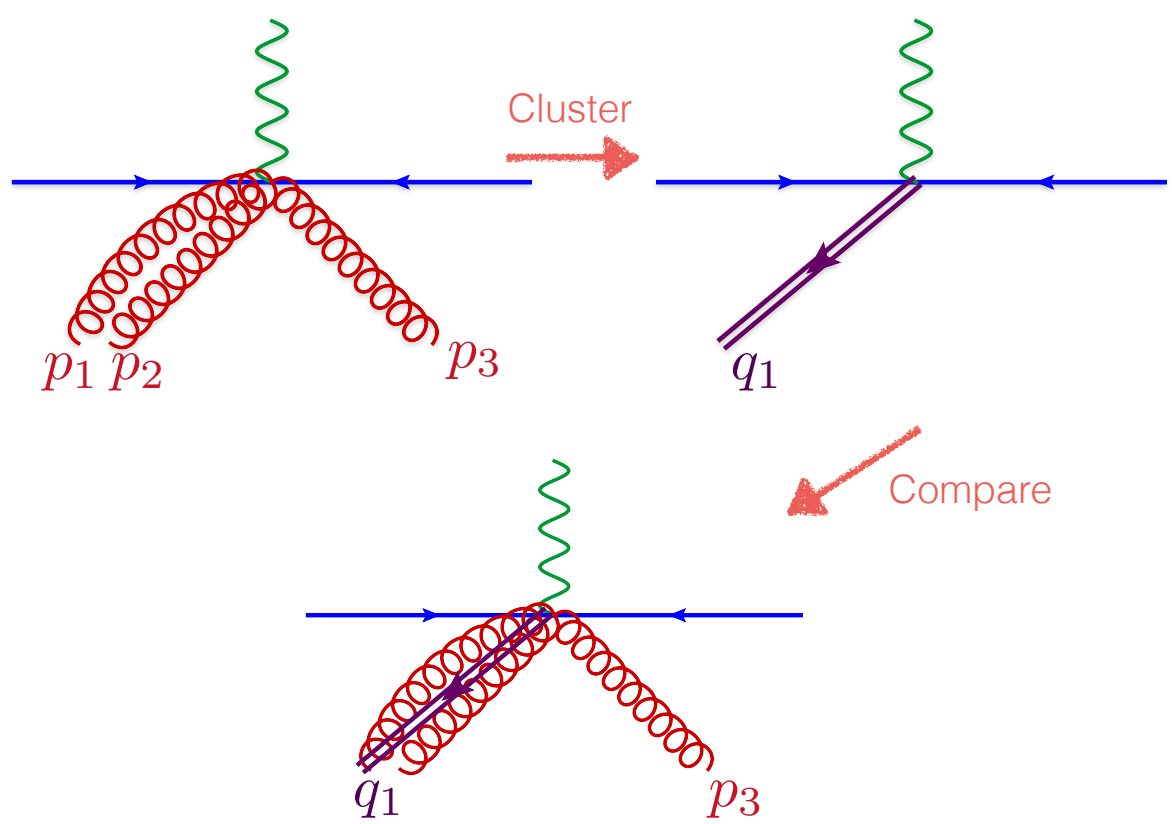

Figure 13: A representation of an event with a larger value of $\mathscr{T}_{1}$, here a wide angle emission forces the invariant $p_{3} \cdot q_{1}$ away from zero, accordingly here only a single unresolved limit is possible.

final state collinear radiation. They are all process independent (although the soft function is a matrix in color space and thus depends on the number and nature of colored scatters). $H$ is a process dependent hard function, which (after suitable definition) is IR finite. This below cut bit then plays the role of $\log \delta \cdot V_{n} F_{n}^{J}$ in our toy model. Here however the fourth powers of the $\log$ occur.

The important feature in any of these slicing calculations is thus an ability to show a lack of sensitivity to your cut-off. This is shown for several color singlet process in Figure 14, which is taken from MCFM [42]. $N$-jettiness slicing has become a popular method for NNLO calculations, and has been applied to multiple LHC processes at NNLO [38, 39, 40, 41, 42, 107, 108, 109]. For color singlet production, $Q_{T}$ slicing [110] has also been widely used [111, 45, 112, 56, 20, 55, 113].

\subsubsection{Subtraction methods}

We can overcome all of the issues described above if instead of approximating the matrix element we simply add in a subtraction term

$$
-R_{n}(0) F_{n}^{J} \int_{0}^{1} \frac{d x}{x^{1+\varepsilon}}
$$

Of course we cannot add terms to the cross section with impunity. If we add it in somewhere we better take it away somewhere else.

$$
\sigma^{(1)}=\frac{V_{n}}{\varepsilon} F_{n}^{J}+R_{n}(0) F_{n}^{J} \int_{0}^{1} \frac{d x}{x^{1+\varepsilon}}+\int_{0}^{1} \frac{d x}{x^{1+\varepsilon}} R_{n}(x) F_{n+1}^{J}(x)-R_{n}(0) F_{n}^{J} \int_{0}^{1} \frac{d x}{x^{1+\varepsilon}}
$$



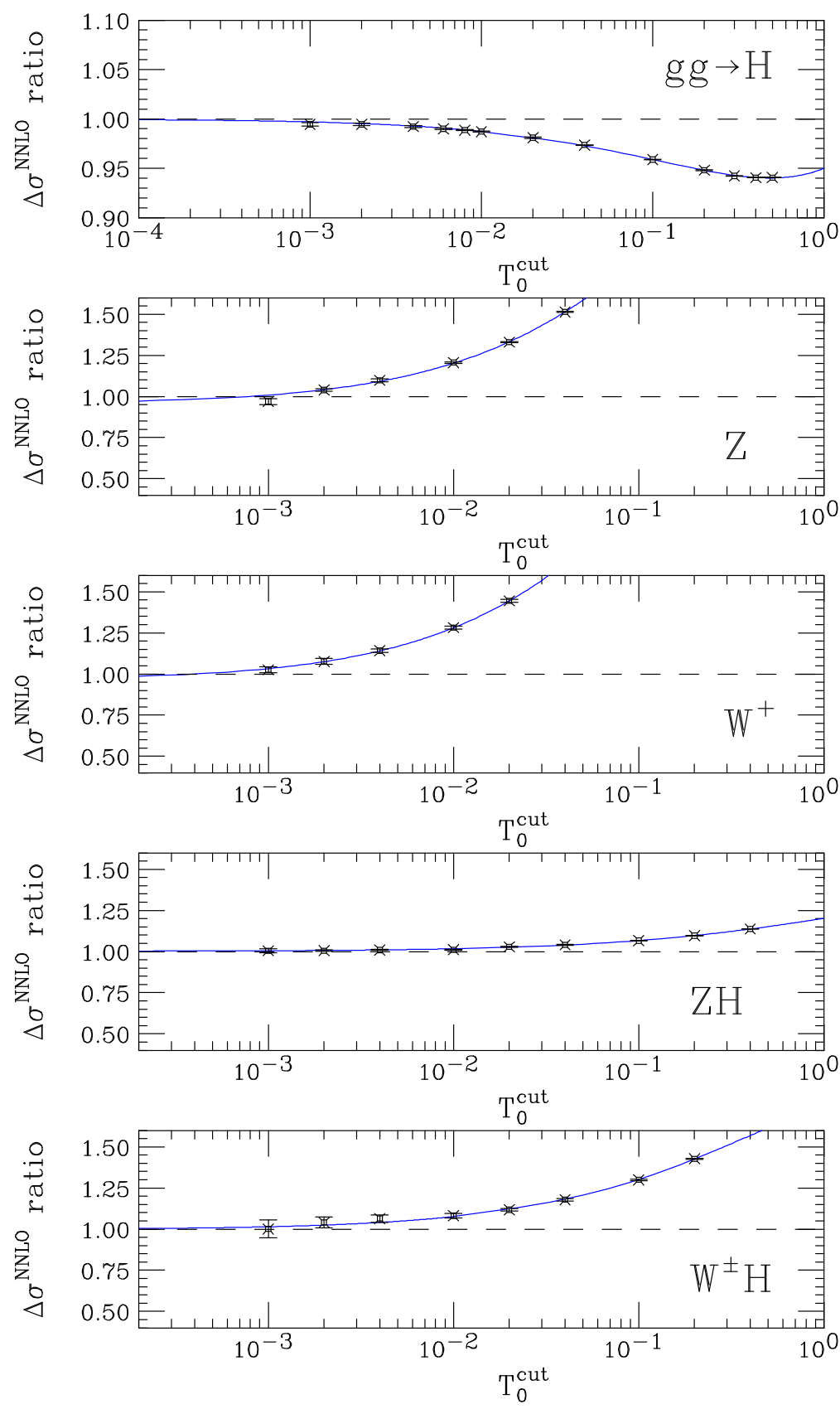

Figure 14: Slicing dependence at NNLO for known processes taken from Ref. [42] 
Now we integrate the first one, and re-package the second,

$$
\sigma^{(1)}=\left(\frac{V_{n}}{\varepsilon}-\frac{R_{n}(0)}{\varepsilon}\right) F_{n}^{J}+\int_{0}^{1} \frac{d x}{x^{1+\varepsilon}}\left[R_{n}(x) F_{n+1}^{J}(x)-R_{n}(0) F_{n}^{J}\right]
$$

Lets look at the second term, as $x \rightarrow 0$.

$$
\lim _{x \rightarrow 0}\left[R_{n}(x) F_{n+1}^{J}(x)-R_{n}(0) F_{n}^{J}\right]=R_{n}(0) F_{n}^{J}(0)+\left.\frac{d R_{n}(x) F_{n+1}^{J}(x)}{d x}\right|_{x=0} x-R_{n}(0) F_{n}^{J}=\mathscr{O}(x)
$$

and as such our integral is finite. Therefore we no longer need $\varepsilon$ as a regulating parameter and we can simply put $\varepsilon=0$ in the second integral. Note again the essential role of the infra-red safety of the measurement function $F_{n+1}^{J}(x)$ in this argument. Again our KLN theorem for the toy model requires that

$$
R_{n}(0)=V_{n}
$$

Such that

$$
\sigma^{(1)}=\int_{0}^{1} \frac{d x}{x}\left[R_{n}(x) F_{n+1}^{J}(x)-V_{n} F_{n}^{J}\right]
$$

Clearly the subtraction method has several advantages over the slicing method outlined previously. Note that a large cancellation still has to take place (between the subtraction term and the real matrix element in the region of $x=0$ ), but if the code is efficiently written this can be performed in such a manner as to result in a stable code (since the cancellation happens point-wise under the integral and not inclusively). The complexity in subtraction methods is the subtractions themselves. Our toy model is not sufficient to demonstrate exactly these complexities.

In general we have to generate a mapping between the real emission phase space and the LO phase space, such that momentum in the LO phase space is physical and factorization can be used. Clearly we cant re-map two particles (emitter and emitted) in such a way as to generate an on-shell combination of the two. We therefore use three particles, (emitter, emitted and spectator) and our mapping thus alters all three such that the combination of emitter and emitted is on-shell and so is the recoiling spectator. We call this a dipole transformation, and the most widespread subtraction at NLO is due to Catani and Seymour [30]. Hence the name Catani-Seymour dipoles. However other schemes exist $[114,115]$ and are used in a variety of different codes, at the end of the day the choice is somewhat arbitrary, any functions which fulfill the requirements above will satisfactorily perform as subtractions.

A further challenge is to ensure that the dipoles can be analytically integrated to cancel the poles in $\varepsilon$ too. At NNLO this is a huge challenge, and the development of a subtraction scheme for LHC calculations was an epic piece of work spread over many years (but now yielding results). The most utilized method at NNLO is antenna subtraction [21], which has successfully been applied to a wide number of LHC processes at NNLO [51, 52, 53, 54, 116, 117, 118].

\section{Putting it all together, an example of $H \rightarrow b \bar{b}$ at NLO}

Lets work all this through in a more meaningful example, we'll look at the decay of a Higgs boson into two $b$ quarks at NLO. This process has been studied both at NNLO and even $\mathrm{N}^{3} \mathrm{LO}$, here 
we'll simply look at the application of a subtraction in action at NLO. Its a useful process since we can see the basic points without worrying about initial state complexities (PDFs) or application of a measurement function (since the process is finite at LO). We therefore simply take $F_{n}^{J}=1$ and look at the application of a counter term in a simple setting. Specifically we consider

$$
H\left(p_{H}\right) \rightarrow b\left(p_{1}\right)+b\left(p_{2}\right)
$$

We'll consider the quarks as massless aside from the Yukawa coupling to the Higgs boson $y_{B}$. The LO process for an on-shell Higgs boson is then simply

$$
\left|\mathscr{M}^{(0)}\right|^{2}=2 N_{c} m_{H}^{2} y_{B}^{2}
$$

To obtain the partial width for example, we would integrate this matrix element over the following two-body phase space (keeping the $d$ dependence for now)

$$
d \Phi_{2}\left(p_{1}, p_{2}\right)=\frac{d^{d} p_{1}}{(2 \pi)^{d-1}} \delta^{(+)}\left(p_{1}^{2}\right) \frac{d^{d} p_{2}}{(2 \pi)^{d-1}} \delta^{(+)}\left(p_{2}^{2}\right)(2 \pi)^{d} \delta^{d}\left(p_{H}-p_{1}-p_{2}\right)
$$

At NLO we will need the real emission process

$$
H\left(p_{H}\right) \rightarrow b\left(p_{1}\right)+b\left(p_{2}\right)+g\left(p_{3}\right)
$$

If we work in $d$-dimensions then the matrix element is proportional to ${ }^{2}$

$$
\left|\mathscr{M}_{H b b g}^{(0)}\right|^{2}=\frac{\alpha_{s}}{2 \pi} 2 m_{H}^{2} N_{c}(2 \pi)^{2} C_{F} \hat{P}_{q q}(x)\left(\frac{1}{s_{13}}+\frac{1}{s_{23}}\right)=\frac{\alpha_{s}}{2 \pi}\left|\mathscr{M}^{(0)}\right|^{2}(2 \pi)^{2} C_{F} \hat{P}_{q q}(x)\left(\frac{1}{s_{13}}+\frac{1}{s_{23}}(\overline{s .5})\right.
$$

where $x=s_{12} / m_{H}^{2}$ and the splitting function is given by

$$
\hat{P}_{q q}(z)=\left(\frac{1+z^{2}}{1-z}-\varepsilon(1-z)\right)
$$

This amplitude is integrated over the three-body phase space

$$
d \Phi_{3}\left(p_{1}, p_{2}, p_{3}\right)=\frac{d^{d} p_{1}}{(2 \pi)^{d-1}} \delta^{(+)}\left(p_{1}^{2}\right) \frac{d^{d} p_{2}}{(2 \pi)^{d-1}}\left(p_{2}^{2}\right) \frac{d^{d} p_{3}}{(2 \pi)^{d-1}} \delta^{(+)}\left(p_{3}^{2}\right)(2 \pi)^{d} \delta^{d}\left(p_{H}-p_{1}-p_{2}-1\left(k_{3}\right), 7\right)
$$

Lets see how we handle this calculation with a dipole subtraction. We have two dipole configurations, one which corresponds to $p_{3}$ being emitted from $p_{1}$ and $p_{2}$ the spectator, and a second dipole with $p_{1}$ and $p_{2}$ swapped. We need to define a mapping back to our LO phase space, so we introduce the following momentum

$$
\begin{aligned}
& \tilde{p}_{13}^{\mu}=p_{1}^{\mu}+p_{3}^{\mu}-\frac{y_{1}}{1-y_{1}} p_{2}^{\mu} \\
& \tilde{p}_{2}^{\mu}=\frac{1}{1-y_{1}} p_{2}^{\mu}
\end{aligned}
$$

where $y_{1}$ is defined as

$$
y_{1}=\frac{s_{13}}{s_{12}+s_{23}+s_{13}}=\frac{s_{13}}{m_{H}^{2}}
$$

\footnotetext{
${ }^{2}$ I've factored out $\left(\mu^{2} e^{\gamma_{E}} / 4 \pi\right)^{\varepsilon}$ into the overall normalization
} 
We see that the $0 \leq y_{1} \leq 1$ defines the range of $y_{1}$. You can see by inserting this value that both $\tilde{p}_{13}$ and $\tilde{p}_{2}$ are on-shell, and that $p_{1}+p_{2}+p_{3}=\tilde{p}_{13}+\tilde{p}_{2}$. We will also need the following variable $z_{1}$

$$
z_{1}=\frac{s_{12}}{s_{23}+s_{12}}
$$

Again the possible range of values of $z_{1}$ are $0 \leq z_{1} \leq 1$. We then construct the following (final-final) Catani-Seymour Dipole [30]

$$
D_{13,2}=-\frac{1}{s_{13}}\left(\frac{2}{1-z_{1}\left(1-y_{1}\right)}-\left(1-z_{1}\right)-\varepsilon\left(1-z_{1}\right)\right)
$$

The second dipole is obtained by swapping $1 \leftrightarrow 2$ everywhere. Our bold claim is then that the following combination

$$
\mathscr{R}=\frac{\alpha_{s}}{2 \pi}\left|\mathscr{M}^{(0)}\right|^{2}(2 \pi)^{2}\left[\hat{P}_{q q}(x)\left(\frac{1}{s_{13}}+\frac{1}{s_{23}}\right)+D_{13,2}+D_{23,1}\right]
$$

is finite as either $s_{13} \rightarrow 0$ or $s_{23} \rightarrow 0$ or $s_{13}$ and $s_{23} \rightarrow 0$ simultaneously. We note that a more general application would apply different measurement functions to the real matrix element and dipoles, we suppressed the measurement function completely in this example, but in general the IR safety requirements will always force the real phase space's measurement function onto the LO (dipole) measurement function, in a singular limit. So checking the above formula is good enough to test the cancellation of soft and collinear singularities. In order to show this we define

$$
\mathscr{R}^{\prime}\left(s_{12}, s_{23}, s_{13}\right)=\left[\hat{P}_{q q}(x)\left(\frac{1}{s_{13}}+\frac{1}{s_{23}}\right)+D_{13,2}+D_{23,1}\right]
$$

Inserting our definitions of $z_{1}$ and $y_{1}$ (and $y_{2}$ and $z_{2}$ ) into the above gives (after some simplification, a handy job for Mathematica)

$$
\mathscr{R}^{\prime}\left(s_{12}, s_{23}, s_{13}\right)=(1-\varepsilon) \frac{s_{12}\left(s_{12}+m_{H}^{2}\right)}{\left(s_{12}+s_{13}\right)\left(s_{12}+s_{23}\right) m_{H}^{2}}
$$

This function is manifestly behaved in all limits, for example

$$
\mathscr{R}^{\prime}\left(s_{12}, 0,0\right)=\frac{2(1-\varepsilon)}{s_{12}}, \quad \mathscr{R}^{\prime}\left(s_{12}, s_{23}, 0\right)=\frac{2(1-\varepsilon)\left(2 s_{12}+s_{23}\right)}{\left(s_{12}+s_{23}\right)^{2}}
$$

and $\mathscr{R}^{\prime}\left(s_{12}, 0, s_{13}\right)$ can be readily obtained by symmetry.

\subsection{Integrating the dipoles}

We have to integrate our dipoles over the unresolved phase space to obtain an analytic expression for the integrated dipoles, which can be used to cancel the remaining poles in the virtual. We can write the three-body phase space given by eq. 5.7 in terms of the two-body phase space for the $\tilde{p}$ momentum and integration over our dipole variable $z_{i}$ and $y_{i}$.

$$
d \Phi_{3}\left(p_{1}, p_{2}, p_{3}\right) \rightarrow d \Phi_{2}\left(\tilde{p}_{13}, \tilde{p}_{2}\right)\left[d p_{i}\left(\tilde{p}_{13}, \tilde{p}_{2}\right)\right]
$$


where

$$
\left[d p_{i}\left(\tilde{p}_{13}, \tilde{p}_{2}\right)\right]=\frac{m_{H}^{2-2 \varepsilon}}{16 \pi^{2}} \frac{d \Omega^{(d-3)}}{(2 \pi)^{1-2 \varepsilon}} d z_{1} d y_{1}\left(z_{1}\left(1-z_{1}\right)\right)^{-\varepsilon}\left(1-y_{1}\right)^{1-2 \varepsilon} y_{1}^{-\varepsilon}
$$

where $d \Omega^{d-3}$ is the element of solid angle given by

$$
\int d \Omega^{d-3}=\frac{2 \pi}{\pi^{\varepsilon} \Gamma(1-\varepsilon)}
$$

We can use this phase space parametrization to define an integrate dipole

$$
\mathscr{D}_{13,2}=\int\left[d p_{i}\left(\tilde{p}_{13}, \tilde{p}_{2}\right)\right]\left(-D_{13,2}\right)
$$

Or, supressing some overall normalization for clarity

$$
\mathscr{D}_{13,2} \propto \int_{0}^{1} d z_{1}\left(z_{1}\left(1-z_{1}\right)\right)^{-\varepsilon} \int_{0}^{1} \frac{d y}{y}(1-y)^{1-2 \varepsilon} y^{-\varepsilon}\left(\frac{2}{1-z_{1}\left(1-y_{1}\right)}-\left(1+z_{1}\right)-\varepsilon\left(1-z_{1}\right)(\bar{j} .21)\right.
$$

Putting this into Mathematica we compute the following result

$$
\mathscr{D}_{13,2} \propto \frac{\Gamma^{3}(1-\varepsilon)}{\Gamma(1-3 \varepsilon)}\left(\frac{1}{\varepsilon^{2}}+\frac{1}{\varepsilon} \frac{3+\varepsilon}{2(1-3 \varepsilon)}\right)
$$

Expanding out the series we find

$$
\mathscr{D}_{13,2} \propto\left(\frac{1}{\varepsilon^{2}}+\frac{3}{2 \varepsilon}+5-\frac{\pi^{2}}{2}+\mathscr{O}(\varepsilon)\right)
$$

Next we turn our attention to the virtual piece, normalized to the $\mathrm{LO}$ (and a factor of $C_{F}$ such that its in the same units as above) we find that

$$
V=B_{2,1}\left(\frac{4}{D-4}+D\right)
$$

where $B_{2,1}$ is the bubble basis integral, expanded in $\varepsilon$ by

$$
B_{2,1}=\frac{1}{\varepsilon}+2+\mathscr{O}(\varepsilon)
$$

Here we have assumed that $\mu^{2}=m_{H}^{2}=s_{12}$.

Expanding our result our we find

$$
V=-\frac{2}{\varepsilon^{2}}+\left(\zeta_{2}-2\right)+\mathscr{O}(\varepsilon)
$$

where $\zeta_{2}=\pi^{2} / 6$. Thus our combined virtual - integrated dipole terms are, again working in units of the LO (and technically evaluating $\alpha_{s}$ at $\mu^{2}=m_{H}^{2}$ we find

$$
T=V+\mathscr{D}_{13,2}+\mathscr{D}_{23,1}=-\frac{2}{\varepsilon^{2}}+\left(\zeta_{2}-2\right)+2\left(\frac{1}{\varepsilon^{2}}+\frac{3}{2 \varepsilon}+5-\frac{\pi^{2}}{2}\right)
$$


Or

$$
T=\frac{3}{\varepsilon}-\frac{5 \pi^{2}}{6}+8
$$

Oh dear, this doesnt look very finite. However we have not performed any renormalization of any UV divergences. Since this the first time $\alpha_{s}$ occurs in the expansion we dont have to renormalize that, on the other hand the Yukawa coupling requires renormalization, which in our massless theory in the $\overline{\mathrm{MS}}$ scheme provides proceeds as follows,

$$
y_{b}^{B}=y_{b}\left[1-\frac{\alpha_{s}}{4 \pi} \frac{3 C_{F}}{\varepsilon}+\ldots\right]
$$

Since our process comes in at $y_{B}^{2}$ the corresponding correction is (in units of $C_{F} \alpha_{s} /(2 \pi)$ as our renormalized piece is

$$
T^{\prime}=T-\frac{3}{\varepsilon}=8-\frac{5 \pi^{2}}{6}
$$

and is finite.

\section{Conclusions}

These lectures notes have covered a slightly eclectic range of topics related to precision calculations at the LHC. The basic idea was to provide a brief overview of methods used in fixed order computations at NLO and NNLO, such that the reader could delve deeper into any topic which took their interest. Precision physics has and will continue to, play a vital role in the ongoing successfully running of the LHC. It is vital that we understand the SM to such a degree as to reliably interpret any suspected deviation from its predictions observed experimentally. Over the next decade (and at any future colliders) the study of the Higgs boson differentially will require astounding precision from the theoretical community to allow us to probe the nature of the Higgs and the mysteries of electroweak symmetry breaking. These notes provided an introduction to precision physics, hopefully placing an emphasis on important topics which are not normally included in traditional graduate lectures on Quantum Field Theory. We investigated the management of multi-particle scattering amplitudes through color and helicity decompositions. We toured the mathematical properties of tree- and loop-level amplitudes, learning about some of the developments in on-shell methods. We discussed the problems associated with IR singularities at higher orders, and presented an overview of how to tackle them in a Monte Carlo implementation using either slicing methods, or subtraction. Finally we presented an application of subtraction methods to a real process, the decay of a Higgs boson to $b \bar{b}$ at NLO.

\section{Acknowledgements}

I'm grateful to Tilman Plehn and Tracy Slatyer for the invitation to present these lectures at TASI 2018, and the students and local organizers at UC Bolder for having such a fantastic time there. I'm grateful to John Campbell, Lance Dixon and Keith Ellis for providing source material for these lectures. 


\section{References}

[1] ATLAS collaboration, Measurement of the inclusive isolated prompt photon cross section in pp collisions at $\sqrt{s}=8 \mathrm{TeV}$ with the ATLAS detector, JHEP 08 (2016) 005 [1 605.03495 ].

[2] ATLAS collaboration, Measurement of the ZZ Production Cross Section in pp Collisions at $\sqrt{s}=$ 13 TeV with the ATLAS Detector, Phys. Rev. Lett. 116 (2016) 101801 [1512.05314].

[3] ATLAS collaboration, Measurement of the $W^{ \pm} Z$ boson pair-production cross section in pp collisions at $\sqrt{s}=13 \mathrm{TeV}$ with the ATLAS Detector, Phys. Lett. B762 (2016) 1 [1606.04017].

[4] ATLAS collaboration, Measurement of the isolated di-photon cross-section in pp collisions at $\sqrt{s}=7$ TeV with the ATLAS detector, Phys. Rev. D85 (2012) 012003 [1107. 0581$].$

[5] ATLAS collaboration, Measurement of total and differential $W^{+} W^{-}$production cross sections in proton-proton collisions at $\sqrt{s}=8 \mathrm{TeV}$ with the ATLAS detector and limits on anomalous triple-gauge-boson couplings, JHEP 09 (2016) 029 [1603.01702].

[6] CMS collaboration, Measurement of the triple-differential cross section for photon+jets production in proton-proton collisions at $\sqrt{s}=7 \mathrm{TeV}$, JHEP 06 (2014) 009 [1311.6141].

[7] CMS collaboration, Measurement of differential cross sections for the production of a pair of isolated photons in pp collisions at $\sqrt{s}=7$ TeV, Eur. Phys. J. C74 (2014) 3129 [1405. 722 5].

[8] CMS collaboration, Measurement of the $W \gamma$ and $Z \gamma$ inclusive cross sections in pp collisions at $\sqrt{s}=7$ TeV and limits on anomalous triple gauge boson couplings, Phys. Rev. D89 (2014) 092005 [1308.6832].

[9] CMS collaboration, Measurement of the $\mathrm{W}^{+} \mathrm{W}^{-}$cross section in pp collisions at $\sqrt{s}=8$ TeV and limits on anomalous gauge couplings, Eur. Phys. J. C76 (2016) 401 [1507. 03268].

[10] ATLAS collaboration, Observation of a new particle in the search for the Standard Model Higgs boson with the ATLAS detector at the LHC, Phys. Lett. B716 (2012) 1 [1207. 7214].

[11] CMS collaboration, Observation of a New Boson at a Mass of $125 \mathrm{GeV}$ with the CMS Experiment at the LHC, Phys. Lett. B716 (2012) 30 [1207. 7235].

[12] R. Contino et al., Physics at a 100 TeV pp collider: Higgs and EW symmetry breaking studies, CERN Yellow Rep. (2017) 255 [1606.09408].

[13] C. Anastasiou, C. Duhr, F. Dulat, F. Herzog and B. Mistlberger, Higgs Boson Gluon-Fusion Production in QCD at Three Loops, Phys. Rev. Lett. 114 (2015) 212001 [1503. 06056 ].

[14] C. Anastasiou, C. Duhr, F. Dulat, E. Furlan, T. Gehrmann, F. Herzog et al., High precision determination of the gluon fusion Higgs boson cross-section at the LHC, JHEP 05 (2016) 058 [1602.00695].

[15] S. Dulat, T.-J. Hou, J. Gao, M. Guzzi, J. Huston, P. Nadolsky et al., New parton distribution functions from a global analysis of quantum chromodynamics, Phys. Rev. D93 (2016) 033006 [1506.07443].

[16] L. A. Harland-Lang, A. D. Martin, P. Motylinski and R. S. Thorne, Parton distributions in the LHC era: MMHT 2014 PDFs, Eur. Phys. J. C75 (2015) 204 [1412. 3989].

[17] NNPDF collaboration, Parton distributions for the LHC Run II, JHEP 04 (2015) 040 [1410.8849]. 
[18] D. Curtin, P. Jaiswal and P. Meade, Charginos Hiding In Plain Sight, Phys. Rev. D87 (2013) 031701 [1206.6888].

[19] J. S. Kim, K. Rolbiecki, K. Sakurai and J. Tattersall, 'Stop' that ambulance! New physics at the LHC?, JHEP 12 (2014) 010 [1406.0858].

[20] M. Grazzini, S. Kallweit, S. Pozzorini, D. Rathlev and M. Wiesemann, $W^{+} W$ production at the LHC: fiducial cross sections and distributions in NNLO QCD, JHEP 08 (2016) 140 [1605 . 02716 ].

[21] A. Gehrmann-De Ridder, T. Gehrmann and E. W. N. Glover, Antenna subtraction at NNLO, JHEP 09 (2005) 056 [hep-ph/ 0505111$].$

[22] M. Czakon, A novel subtraction scheme for double-real radiation at NNLO, Phys. Lett. $\mathbf{B 6 9 3}$ (2010) $259[1005.0274]$.

[23] M. Czakon, Double-real radiation in hadronic top quark pair production as a proof of a certain concept, Nucl. Phys. B849 (2011) 250 [1101.0642].

[24] R. Boughezal, C. Focke, X. Liu and F. Petriello, $W$-boson production in association with a jet at next-to-next-to-leading order in perturbative QCD, Phys. Rev. Lett. 115 (2015) 062002 [1504.02131].

[25] J. Gaunt, M. Stahlhofen, F. J. Tackmann and J. R. Walsh, N-jettiness Subtractions for NNLO QCD Calculations, JHEP 09 (2015) 058 [1505. 04794].

[26] M. Cacciari, F. A. Dreyer, A. Karlberg, G. P. Salam and G. Zanderighi, Fully Differential Vector-Boson-Fusion Higgs Production at Next-to-Next-to-Leading Order, Phys. Rev. Lett. 115 (2015) 082002 [1506.02660].

[27] W. T. Giele and E. W. N. Glover, Higher order corrections to jet cross-sections in e+e-annihilation, Phys. Rev. D46 (1992) 1980.

[28] W. T. Giele, E. W. N. Glover and D. A. Kosower, Higher order corrections to jet cross-sections in hadron colliders, Nucl. Phys. B403 (1993) 633 [hep-ph/9302225].

[29] I. W. Stewart, F. J. Tackmann and W. J. Waalewijn, N-Jettiness: An Inclusive Event Shape to Veto Jets, Phys. Rev. Lett. 105 (2010) 092002 [1004.2489].

[30] S. Catani and M. H. Seymour, A General algorithm for calculating jet cross-sections in NLO QCD, Nucl. Phys. B485 (1997) 291 [hep-ph/9605323].

[31] I. W. Stewart, F. J. Tackmann and W. J. Waalewijn, Factorization at the LHC: From PDFs to Initial State Jets, Phys. Rev. D81 (2010) 094035 [0910 . 0467$].$

[32] I. W. Stewart, F. J. Tackmann and W. J. Waalewijn, The Beam Thrust Cross Section for Drell-Yan at NNLL Order, Phys. Rev. Lett. 106 (2011) 032001 [1005.4060].

[33] C. W. Bauer, S. Fleming and M. E. Luke, Summing Sudakov logarithms in B $\rightarrow X($ s gamma) in effective field theory, Phys. Rev. D63 (2000) 014006 [hep-ph / 0005275 ].

[34] C. W. Bauer, S. Fleming, D. Pirjol and I. W. Stewart, An Effective field theory for collinear and soft gluons: Heavy to light decays, Phys. Rev. D63 (2001) 114020 [hep-ph / 0011336 ].

[35] C. W. Bauer and I. W. Stewart, Invariant operators in collinear effective theory, Phys. Lett. B516 (2001) 134 [hep-ph/0107001].

[36] C. W. Bauer, D. Pirjol and I. W. Stewart, Soft collinear factorization in effective field theory, Phys. Rev. D65 (2002) 054022 [hep-ph/ 0109045$].$ 
[37] C. W. Bauer, S. Fleming, D. Pirjol, I. Z. Rothstein and I. W. Stewart, Hard scattering factorization from effective field theory, Phys. Rev. D66 (2002) 014017 [hep-ph/ 0202088 ].

[38] R. Boughezal, C. Focke, W. Giele, X. Liu and F. Petriello, Higgs boson production in association with a jet at NNLO using jettiness subtraction, Phys. Lett. B748 (2015) 5 [1505 . 03893 ].

[39] R. Boughezal, J. M. Campbell, R. K. Ellis, C. Focke, W. T. Giele, X. Liu et al., Z-boson production in association with a jet at next-to-next-to-leading order in perturbative QCD, Phys. Rev. Lett. 116 (2016) 152001 [1512.01291].

[40] J. M. Campbell, R. K. Ellis and C. Williams, Associated production of a Higgs boson at NNLO, JHEP 06 (2016) 179 [1601.00658].

[41] J. M. Campbell, R. K. Ellis, Y. Li and C. Williams, Predictions for diphoton production at the LHC through NNLO in QCD, JHEP 07 (2016) 148 [1603.02663].

[42] R. Boughezal, J. M. Campbell, R. K. Ellis, C. Focke, W. Giele, X. Liu et al., Color singlet production at NNLO in MCFM, Eur. Phys. J. C77 (2017) 7 [1605.08011].

[43] G. Ferrera, M. Grazzini and F. Tramontano, Associated WH production at hadron colliders: a fully exclusive QCD calculation at NNLO, Phys. Rev. Lett. 107 (2011) 152003 [1107.1164].

[44] F. Cascioli, T. Gehrmann, M. Grazzini, S. Kallweit, P. MaierhÃúfer, A. von Manteuffel et al., ZZ production at hadron colliders in NNLO QCD, Phys. Lett. B735 (2014) 311 [1405 .2219].

[45] M. Grazzini, S. Kallweit and D. Rathlev, Wr and Z $\gamma$ production at the LHC in NNLO QCD, JHEP 07 (2015) 085 [1504.01330].

[46] M. Czakon and A. Mitov, NNLO corrections to top-pair production at hadron colliders: the all-fermionic scattering channels, JHEP 12 (2012) 054 [1207. 0236].

[47] M. Czakon and A. Mitov, NNLO corrections to top pair production at hadron colliders: the quark-gluon reaction, JHEP 01 (2013) 080 [1210.6832].

[48] M. Czakon, P. Fiedler and A. Mitov, Total Top-Quark Pair-Production Cross Section at Hadron Colliders Through $O\left(\frac{4}{S}\right)$, Phys. Rev. Lett. 110 (2013) 252004 [1303. 62 54].

[49] R. Boughezal, F. Caola, K. Melnikov, F. Petriello and M. Schulze, Higgs boson production in association with a jet at next-to-next-to-leading order in perturbative QCD, JHEP 06 (2013) 072 [1302.6216].

[50] R. Boughezal, F. Caola, K. Melnikov, F. Petriello and M. Schulze, Higgs boson production in association with a jet at next-to-next-to-leading order, Phys. Rev. Lett. 115 (2015) 082003 [1504.07922].

[51] A. Gehrmann-De Ridder, T. Gehrmann, E. W. N. Glover and J. Pires, Second order QCD corrections to jet production at hadron colliders: the all-gluon contribution, Phys. Rev. Lett. 110 (2013) 162003 [1301.7310].

[52] X. Chen, T. Gehrmann, E. W. N. Glover and M. Jaquier, Precise QCD predictions for the production of Higgs + jet final states, Phys. Lett. B740 (2015) 147 [1408.532 5].

[53] A. Gehrmann-De Ridder, T. Gehrmann, E. W. N. Glover, A. Huss and T. A. Morgan, Precise QCD predictions for the production of a $Z$ boson in association with a hadronic jet, Phys. Rev. Lett. 117 (2016) 022001 [1507.02850].

[54] A. Gehrmann-De Ridder, T. Gehrmann, E. W. N. Glover, A. Huss and T. A. Morgan, The NNLO QCD corrections to Z boson production at large transverse momentum, JHEP 07 (2016) 133 [1605.04295]. 
[55] D. de Florian, M. Grazzini, C. Hanga, S. Kallweit, J. M. Lindert, P. MaierhÃúfer et al., Differential Higgs Boson Pair Production at Next-to-Next-to-Leading Order in QCD, JHEP 09 (2016) 151 [1606.09519].

[56] M. Grazzini, S. Kallweit, D. Rathlev and M. Wiesemann, $W^{ \pm} Z$ production at hadron colliders in NNLO QCD, Phys. Lett. B761 (2016) 179 [1604.08576].

[57] R. K. Ellis, Z. Kunszt, K. Melnikov and G. Zanderighi, One-loop calculations in quantum field theory: from Feynman diagrams to unitarity cuts, Phys. Rept. 518 (2012) 141 [1105.4319].

[58] J. Campbell, Perturbative QCD and NLO Monte Carlo Simulations (TASI 2011), in The Dark Secrets of the Terascale: Proceedings, TASI 2011, Boulder, Colorado, USA, Jun 6 - Jul 11, 2011, pp. 107-159, 2013, DOI.

[59] J. Campbell, J. Huston and F. Krauss, The Black Book of Quantum Chromodynamics. Oxford University Press, 2017.

[60] L. J. Dixon, Calculating scattering amplitudes efficiently, in QCD and beyond. Proceedings, Theoretical Advanced Study Institute in Elementary Particle Physics, TASI-95, Boulder, USA, June 4-30, 1995, pp. 539-584, 1996, hep-ph/9601359, http://www-public.slac.stanford.edu/sciDoc/docMeta.aspx?slacPubNumber=SLAC-PUB-7106.

[61] L. J. Dixon, A brief introduction to modern amplitude methods, in Proceedings, 2012 European School of High-Energy Physics (ESHEP 2012): La Pommeraye, Anjou, France, June 06-19, 2012, pp. 31-67, 2014, 1310.5353 , DOI.

[62] L. J. Dixon, A Brief Introduction to Modern Amplitude Methods, in Proceedings, Theoretical Advanced Study Institute in Elementary Particle Physics: Journeys Through the Precision Frontier: Amplitudes for Colliders (TASI 2014): Boulder, Colorado, June 2-27, 2014, pp. 39-97, 2015, DOI.

[63] J. Alwall, P. Demin, S. de Visscher, R. Frederix, M. Herquet, F. Maltoni et al., MadGraph/MadEvent v4: The New Web Generation, JHEP 09 (2007) 028 [0 706.2334 ].

[64] J. Alwall, M. Herquet, F. Maltoni, O. Mattelaer and T. Stelzer, MadGraph 5 : Going Beyond, JHEP 06 (2011) 128 [1106.0522].

[65] T. Gleisberg, S. Hoeche, F. Krauss, M. Schonherr, S. Schumann, F. Siegert et al., Event generation with SHERPA 1.1, JHEP 02 (2009) 007 [0811. 4622].

[66] M. L. Mangano, M. Moretti, F. Piccinini, R. Pittau and A. D. Polosa, ALPGEN, a generator for hard multiparton processes in hadronic collisions, JHEP 07 (2003) 001 [hep-ph / 0206293 ].

[67] T. Sjostrand, S. Mrenna and P. Z. Skands, PYTHIA 6.4 Physics and Manual, JHEP 05 (2006) 026 [hep-ph/0603175].

[68] T. Sjostrand, S. Mrenna and P. Z. Skands, A Brief Introduction to PYTHIA 8.1, Comput. Phys. Commun. 178 (2008) 852 [0710.3820].

[69] G. Corcella, I. G. Knowles, G. Marchesini, S. Moretti, K. Odagiri, P. Richardson et al., HERWIG 6: An Event generator for hadron emission reactions with interfering gluons (including supersymmetric processes), JHEP 01 (2001) 010 [hep-ph/0011363].

[70] M. Bahr et al., Herwig++ Physics and Manual, Eur. Phys. J. C58 (2008) 639 [0803.0883].

[71] J. Alwall, R. Frederix, S. Frixione, V. Hirschi, F. Maltoni, O. Mattelaer et al., The automated computation of tree-level and next-to-leading order differential cross sections, and their matching to parton shower simulations, JHEP 07 (2014) 079 [1405.0301]. 
[72] T. Gleisberg and S. Hoeche, Comix, a new matrix element generator, JHEP 12 (2008) 039 [0808.3674].

[73] G. 't Hooft, A Planar Diagram Theory for Strong Interactions, Nucl. Phys. B72 (1974) 461.

[74] F. A. Berends and W. Giele, The Six Gluon Process as an Example of Weyl-Van Der Waerden Spinor Calculus, Nucl. Phys. B294 (1987) 700.

[75] M. L. Mangano, The Color Structure of Gluon Emission, Nucl. Phys. B309 (1988) 461.

[76] M. L. Mangano and S. J. Parke, Multiparton amplitudes in gauge theories, Phys. Rept. 200 (1991) 301 [hep-th/0509223].

[77] Z. Bern and D. A. Kosower, Color decomposition of one loop amplitudes in gauge theories, Nucl. Phys. B362 (1991) 389.

[78] Z. Bern, L. J. Dixon and D. A. Kosower, One loop amplitudes for e+e-to four partons, Nucl. Phys. B513 (1998) 3 [hep-ph/9708239].

[79] R. Britto, F. Cachazo and B. Feng, New recursion relations for tree amplitudes of gluons, Nucl. Phys. B715 (2005) 499 [hep-th/ 0412308$].$

[80] R. Britto, F. Cachazo, B. Feng and E. Witten, Direct proof of tree-level recursion relation in Yang-Mills theory, Phys. Rev. Lett. 94 (2005) 181602 [hep-th/ 0501052 ].

[81] N. Arkani-Hamed and J. Kaplan, On Tree Amplitudes in Gauge Theory and Gravity, JHEP 04 (2008) 076 [0801.2385].

[82] A. Brandhuber, P. Heslop and G. Travaglini, A Note on dual superconformal symmetry of the $N=4$ super Yang-Mills S-matrix, Phys. Rev. D78 (2008) 125005 [0807.4097].

[83] J. M. Drummond and J. M. Henn, All tree-level amplitudes in N=4 SYM, JHEP 04 (2009) 018 [0808.2475].

[84] S. D. Badger, E. W. N. Glover, V. V. Khoze and P. Svrcek, Recursion relations for gauge theory amplitudes with massive particles, JHEP 07 (2005) 025 [hep-th/ 050415 9].

[85] L. J. Dixon, J. M. Henn, J. Plefka and T. Schuster, All tree-level amplitudes in massless QCD, JHEP 01 (2011) 035 [1010.3991].

[86] C. F. Berger, Z. Bern, L. J. Dixon, F. Febres Cordero, D. Forde, H. Ita et al., An Automated Implementation of On-Shell Methods for One-Loop Amplitudes, Phys. Rev. D78 (2008) 036003 [0803.4180].

[87] G. Cullen, N. Greiner, G. Heinrich, G. Luisoni, P. Mastrolia, G. Ossola et al., Automated One-Loop Calculations with GoSam, Eur. Phys. J. C72 (2012) 1889 [1111.2034].

[88] F. Cascioli, P. Maierhofer and S. Pozzorini, Scattering Amplitudes with Open Loops, Phys. Rev. Lett. 108 (2012) 111601 [1111.5206].

[89] Z. Bern, L. J. Dixon and D. A. Kosower, Dimensionally regulated pentagon integrals, Nucl. Phys. B412 (1994) 751 [hep-ph/9306240].

[90] R. J. Eden, P. V. Landshoff, D. I. Olive and J. C. Polkinghorne, The analytic s-matrix, .

[91] Z. Bern, L. J. Dixon, D. C. Dunbar and D. A. Kosower, Fusing gauge theory tree amplitudes into loop amplitudes, Nucl. Phys. B435 (1995) 59 [hep-ph/9409265].

[92] Z. Bern, L. J. Dixon, D. C. Dunbar and D. A. Kosower, One loop n point gauge theory amplitudes, unitarity and collinear limits, Nucl. Phys. B425 (1994) 217 [hep-ph/9403226]. 
[93] Z. Bern, L. J. Dixon and D. A. Kosower, One loop corrections to five gluon amplitudes, Phys. Rev. Lett. 70 (1993) 2677 [hep-ph/9302280].

[94] Z. Bern, L. J. Dixon and D. A. Kosower, One loop corrections to two quark three gluon amplitudes, Nucl. Phys. B437 (1995) 259 [hep-ph/9409393].

[95] R. Britto, F. Cachazo and B. Feng, Generalized unitarity and one-loop amplitudes in N=4 super-Yang-Mills, Nucl. Phys. B725 (2005) 275 [hep-th/ 0412103 ].

[96] R. Britto, F. Cachazo and B. Feng, Computing one-loop amplitudes from the holomorphic anomaly of unitarity cuts, Phys. Rev. D71 (2005) 025012 [hep-th/ 0410179 ].

[97] R. Britto, B. Feng and P. Mastrolia, The Cut-constructible part of QCD amplitudes, Phys. Rev. D73 (2006) 105004 [hep-ph/0602178].

[98] C. Anastasiou, R. Britto, B. Feng, Z. Kunszt and P. Mastrolia, D-dimensional unitarity cut method, Phys. Lett. B645 (2007) 213 [hep-ph/0609191].

[99] D. Forde, Direct extraction of one-loop integral coefficients, Phys. Rev. D75 (2007) 125019 [0704.1835].

[100] P. Mastrolia, Double-Cut of Scattering Amplitudes and Stokes' Theorem, Phys. Lett. B678 (2009) $246[0905.2909]$.

[101] S. D. Badger, Direct Extraction Of One Loop Rational Terms, JHEP 01 (2009) 049 [0806. 4600 ].

[102] E. W. Nigel Glover and C. Williams, One-Loop Gluonic Amplitudes from Single Unitarity Cuts, JHEP 12 (2008) 067 [0810.2964].

[103] R. Boughezal, X. Liu and F. Petriello, N-jettiness soft function at next-to-next-to-leading order, Phys. Rev. D91 (2015) 094035 [1504.02540].

[104] J. M. Campbell, R. K. Ellis, R. Mondini and C. Williams, The NNLO QCD soft function for 1-jettiness, Eur. Phys. J. C78 (2018) 234 [1711.09984].

[105] T. Becher and M. Neubert, Toward a NNLO calculation of the anti-B $\rightarrow X(s)$ gamma decay rate with a cut on photon energy. II. Two-loop result for the jet function, Phys. Lett. B637 (2006) 251 [hep-ph/0603140].

[106] T. Becher and G. Bell, The gluon jet function at two-loop order, Phys. Lett. B695 (2011) 252 [1008.1936].

[107] J. M. Campbell, R. K. Ellis and S. Seth, H+1 jet production revisited, 1906.01020.

[108] J. M. Campbell, R. K. Ellis and C. Williams, Direct Photon Production at Next-toâĂŞNext-to-Leading Order, Phys. Rev. Lett. 118 (2017) 222001 [1612 . 04333 ].

[109] J. M. Campbell, T. Neumann and C. Williams, Z $\gamma$ Production at NNLO Including Anomalous Couplings, JHEP 11 (2017) 150 [1708.02925].

[110] S. Catani and M. Grazzini, An NNLO subtraction formalism in hadron collisions and its application to Higgs boson production at the LHC, Phys. Rev. Lett. 98 (2007) 222002 [hep-ph/ 0703012 ].

[111] T. Gehrmann, M. Grazzini, S. Kallweit, P. Maierhãuufer, A. von Manteuffel, S. Pozzorini et al., $W^{+} W^{-}$Production at Hadron Colliders in Next to Next to Leading Order QCD, Phys. Rev. Lett. 113 (2014) 212001 [1408.5243].

[112] M. Grazzini, S. Kallweit and D. Rathlev, ZZ production at the LHC: fiducial cross sections and distributions in NNLO QCD, Phys. Lett. B750 (2015) 407 [1507. 06257 ]. 
[113] M. Grazzini, S. Kallweit, D. Rathlev and M. Wiesemann, $W^{ \pm} Z$ production at the LHC: fiducial cross sections and distributions in NNLO QCD, JHEP 05 (2017) 139 [1703 . 09065 ].

[114] S. Frixione, Z. Kunszt and A. Signer, Three jet cross-sections to next-to-leading order, Nucl. Phys. B467 (1996) 399 [hep-ph/9512328].

[115] Z. Nagy and D. E. Soper, General subtraction method for numerical calculation of one loop QCD matrix elements, JHEP 09 (2003) 055 [hep-ph/ 0308127 ].

[116] J. Currie, A. Gehrmann-De Ridder, T. Gehrmann, E. W. N. Glover, A. Huss and J. Pires, Precise predictions for dijet production at the LHC, Phys. Rev. Lett. 119 (2017) 152001 [1705. 10271 ].

[117] J. Cruz-Martinez, T. Gehrmann, E. W. N. Glover and A. Huss, Second-order QCD effects in Higgs boson production through vector boson fusion, Phys. Lett. B781 (2018) 672 [1802 .024 45].

[118] X. Chen, T. Gehrmann, N. Glover, M. HÃuufer and A. Huss, Isolated photon and photon+jet production at NNLO QCD accuracy, Submitted to: J. High Energy Phys. (2019) [1904.01044]. 